\title{
Automatic Computing Methods for Special Functions. Part IV. Complex Error Function, Fresnel Integrals, and Other Related Functions
}

\author{
Irene A. Stegun* and Ruth Zucker* \\ National Bureau of Standards, Washington, DC 20234
}

July 15,1981

\begin{abstract}
Accurate, efficient, automatic methods for computing the complex error function to any precision are detailed and implemented in an American Standard FORTRAN subroutine. A six significant figure table of erfc $z$, $e^{x^{2}}$ erfc $z$, and $e^{z^{2}}$ erfc $(-z)$ is included for $z$ in polar coordinate form with the modulus of $z$ ranging from 0 to 9. The argand diagram is given for erf $z$.
\end{abstract}

Key words: Argand diagram; complex error function; continued fraction; Dawson's function; FORTRAN subroutine; Fresnel integrals; key values; line broadening function; plasma dispersion function; Voigt function.

\section{Introduction}

In computing many of the functions of mathematical physics, for example, Fresnel integrals, Dawson's integral, Voigt function, plasma dispersion function, etc., difficulties are frequently encountered. Since these functions may be expressed in terms of the error function of complex argument, we have chosen this function for Part IV. ${ }^{1}$ The major part of the coding of the power series, continued fraction and asymptotic expansion computations for complex arguments will carry over equally well for other functions.

As Part I was devoted to the error function of a real variable, the probability function and other related functions, Part IV will only emphasize those functions and pitfalls due to complex arguments.

While accuracy over the entire domain of definition remains our main concern, the methods employed ensure efficiency, portability and ease of programming and modification.

If one supplies approximate values for the maximum machine value, minimum machine value, the upper bound of the sine, cosine routine, and the upper bound to the acceptable relative error and gives the square root of $\pi$ to the required number of significant figures, the detailed methods will work for computations ranging from very low precision to multi-precision.

The argand diagram of erf $z$ is included as well as the implementing ANS FORTRAN program and a six significant figure table of $\operatorname{erfc} z, e^{z^{2}} \operatorname{erfc} z$ and $e^{z^{2}} \operatorname{erfc}(-z)$ for $z$ in polar coordinate form with the modulus of $z$ ranging from 0 to 9.

\section{Mathematical properties}

Relevant formulas are collected here for completeness and ease of reference. In keeping with the convention of the Handbook $[1],{ }^{2} z=x+i y$ is a complex variable.

- Mathematical Analysis Division, Center for Applied Mathematics.

${ }^{1}$ Part 1. Error, Probability, and Related Functions. J. Res. Nat. Bur. Stand. (U.S.). 74(3); 211-224; 1970. Part II. The Exponential Integral Es (x). J. Res. Nat. Bur. Stand. (U.S.). 78(4): 199-216; 1974. Part III. The Sine, Cosine, Exponential Integrals, and Related Functions. J. Res. Nat. Bur. Stani. (U.S.). 80(2): $291-311 ; 1976$.

2 Figures in brackets indicate literature references at the end of this paper. 


\section{A. Definitions}

$$
\begin{gathered}
\operatorname{erf} z=\frac{2}{\sqrt{\pi}} \int_{0}^{z} e^{-t^{2}} d t \\
\operatorname{erfc} z=\frac{2}{\sqrt{\pi}} \int_{z}^{\infty} e^{-t^{2}} d t=1-\operatorname{erf} z
\end{gathered}
$$

(The path of integration is subject to the restriction $\arg t \rightarrow \alpha$ with $|\alpha|<\frac{\pi}{4}$ as $t \rightarrow \infty$ along the path. If $R t^{2}$ remains bounded to the left, $\alpha=\frac{\pi}{4}$ is permissible.)

$$
\begin{aligned}
w(z) & =e^{-z^{2}}\left(1+\frac{2 i}{\sqrt{\pi}} \int_{0}^{z} e^{t^{2}} d t\right)=e^{-z^{2}} \operatorname{erfc}(-i z)=e^{\xi^{2}} \operatorname{erfc} \xi(\xi=-i z) \\
& =\frac{i}{\pi} \int_{-\infty}^{\infty} \frac{e^{-t^{2}} d t}{z-t}=\frac{2 i z}{\pi} \int_{0}^{\infty} \frac{e^{-t^{2}} d t}{z^{2}-t^{2}}(I z>0)
\end{aligned}
$$

with $\quad F(z)=e^{-z^{2}} \int_{0}^{z} e^{t^{2}} d t \quad$ (Dawson's Function)

and $\quad Z(z)=\frac{1}{\sqrt{\pi}} \int_{-\infty}^{\infty} \frac{e^{-t^{2}} d t}{t-z} \quad$ (Plasma Dispersion Function)

$$
\begin{gathered}
E(z)=C(z)+i S(z)=\int_{0}^{z} e^{i \pi x^{3} / 2} d t=\int_{0}^{x} \cos \left(\frac{\pi t^{2}}{2}\right) d t+i \int_{0}^{x} \sin \left(\frac{\pi t^{2}}{2}\right) d t \\
=\frac{1+i}{2} \operatorname{erf}\left[\frac{\sqrt{ } \pi}{2}(1-i) z\right] \quad \text { (Fresnel Functions) } \\
W(x, t)=\frac{\omega}{(4 \pi t)^{1 / 2}} \int_{-\infty}^{\infty} \frac{e^{-u^{2}} d u}{u^{2}+\omega^{2}}=\left(\frac{\pi}{4 t}\right)^{1 / 2} e^{\omega^{2}} \operatorname{erfc} \omega
\end{gathered}
$$

where

$$
\begin{aligned}
& \omega=(1-i x) / 2 t^{1 / 2} \\
& =U(x, t)+i V(x, t) \quad \text { (Voigt Function) } \\
& =\frac{1}{(4 \pi t)^{1 / 2}} \int_{-\infty}^{\infty} \frac{e^{-(x-y)^{2} / 4 t}}{1+y^{2}} d y+\frac{i}{(4 \pi t)^{1 / 2}} \int_{-\infty}^{\infty} \frac{y e^{-(x-y)^{2} / 4 t}}{1+y^{2}} d y \\
& \mathrm{H}(a, u)=\frac{a}{\pi} \int_{-\infty}^{\infty} \frac{e^{-t^{2}} d t}{(u-t)^{2}+a^{2}}=\frac{1}{a \sqrt{\pi}} U\left(u / a, 1 / 4 a^{2}\right) \quad \text { (Line Broadening Function) } \\
& \text { B. Series Expansions } \\
& \begin{array}{l}
\operatorname{erf} z=\frac{2}{\sqrt{ } \pi} \sum_{n=0}^{\infty} \frac{(-1)^{n} z^{2 n+1}}{n !(2 n+1)} \\
=\frac{2}{\sqrt{ } \pi} e^{-x^{2}} \sum_{n=0}^{\infty} \frac{2^{n} z^{2 n+1}}{1 \cdot 3 . .(2 n+1)}
\end{array} \\
& \text { C. Continued Fraction }(R z>0) \\
& e^{z^{2}} \text { erfc } z=\frac{1}{\sqrt{\pi}}\left[\frac{1}{z+} \frac{1 / 2}{z+} \frac{1}{z+} \frac{3 / 2}{z+} \frac{2}{z+} \ldots\right] \\
& =\frac{2 z}{\sqrt{\pi}}\left[\frac{1}{2 z^{2}+1}-\frac{1 \cdot 2}{2 z^{2}+5}-\frac{3 \cdot 4}{2 z^{2}+9}-\cdots\right] \text { ("Even" Form) }
\end{aligned}
$$




\section{Asymptotic Expansion}

$$
e^{z^{2}} \operatorname{erfc} z \sim \frac{1}{\sqrt{\pi}}\left[\frac{1}{z}+\sum_{n=1}^{\infty} \frac{(-1)^{n} 1 \cdot 3 \ldots(2 n-1)}{z\left(2 z^{2}\right)^{n}}\right]\left(z-\infty,|\arg z|<\frac{3 \pi}{4}\right)
$$

\section{E. Symmetry Relations}

$$
\begin{aligned}
\operatorname{erf}(-z) & =-\operatorname{erf} z \\
\operatorname{erf} \bar{z} & =\overline{\operatorname{erf} z} \\
w(-z) & =2 e^{-z^{2}}-w(z) \\
w \overline{(z)} & =\overline{w(-z)} \\
C(-z) & =-C(z), S(-z)=-S(z) \\
C(i z) & =i C(z), S(i z)=-i S(z) \\
C \overline{(z)} & =\overline{C(z)}, S \overline{(z)}=\overline{S(z)}
\end{aligned}
$$

\section{Method}

The main functions under consideration are the error function ERFZ, the complementary error function ERFCZ, and the exponential of $z^{2}$ times the complementary error function EZ2CZ. All other functions may be obtained from these three. To simplify testing, computations are performed for $z$ in the first quadrant $A Z$ and symmetry relations are then employed to make adjustments for other quadrants. For the special case $z$ $=0$, no computations are performed and the following function values are returned: $\mathrm{ERFZ}=0, \mathrm{ERFCZ}=$ 1 and $\mathrm{EZ2CZ}=1$.

Real type variables are used throughout to readily allow for double precision computation if greater accuracy is needed. The machine dependent constants are placed in a labeled section at the beginning of the subroutine. Function references are likewise grouped together when possible and attention called to the statement labels of the remaining function references. Real and imaginary parts of complex variables have $\mathrm{R}$ and $\mathrm{I}$ as final characters.

Since EZ2CZ for $z$ in the first quadrant is machine representable even with the real and imaginary parts of $z$ equal to the maximum machine value CMAX (provided its reciprocal is larger than the minimum machine value CMIN), checking for the range of the argument $z$ has been omitted. However, the extensive range necessitates a fair amount of testing for overflows. Underflows are assumed to be set to zero. Overflows are set equal to the maximum machine value and an error indicator IERR set for the number of functions affected. If only EZ2CZ lies outside the machine range, IERR $=1$, otherwise IERR $=3$. As often as possible, computations are arranged so as to give the correct results for the three functions if they lie within the range of the machine.

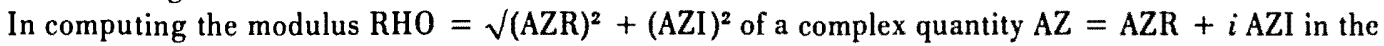
first quadrant, RHO may lie in the machine range but $(A Z R)^{2},(A Z I)^{2}$ or their sum may be outside the range. We select the larger ARIMX and smaller ARIMN of either AZR or AZI, and compute the ratio RMNMX = ARIMN/ARIMX. A factor of RHO called PRHO is computed as the square root of (RMNMX*RMNMX + $\mathrm{ONE}$ ). This factor, which is greater than or equal to one and less than or equal to the square root of 2 , can then be used to check for overflow. The quantity ARIMX must be less than CMAX divided by PRHO for $\mathrm{RHO}$ to lie in the machine range. A similar procedure is followed in computing the real and imaginary parts of $(\mathrm{AZ})^{2}=Z 2 \mathrm{R}+i \mathrm{AZ2I}=(\mathrm{AZR})^{2}-(\mathrm{AZI})^{2}+i 2 \mathrm{AZR}^{*} \mathrm{AZI}$ with first checking to ensure ARIMX is greater than or equal to 1 .

Analysis has indicated and testing confirmed that the power series PS is most useful from the standpoint of accuracy and efficiency for RHO less than RHOLS $(=1.5)$ and when AZR is less than or equal to 1 provid. 
ed RHO is less than AELL $(=\sqrt{ }-\ln ($ TOLER $)$ ) where TOLER is the upper limit for the relative error. The continued fraction expansion CF is most useful for AZR greater than 1 and RHO greater than or equal to RHOLS. The asymptotic expansion AE is most useful for AZR less than or equal to 1 for RHO greater than or equal to AELL. For RHO greater than or equal to RHOLC $(=\sqrt{ } 0.5 / T O L E R)$ a rearrangement of arithmetic operations for the first term of the asymptotic expansion is necessary to maintain the accuracy of EZ2CZ. In the continued fraction and asymptotic expansion regions only, EZ2CZ is first computed; it tends to zero for large $|z|$ and the exponential of $-z^{2}$ tends to infinity for small AZR. To maintain accuracy here, we compute the exponential of $-\mathrm{Z} 2 \mathrm{R} / 6$ and do continuous multiplication and testing with appropriate factors to obtain ERFC. The imaginary part of $(\mathrm{AZ})^{2}$ is tested against ULSC, the upper limit of the sine, cosine routine.

Figure 1 below maps the regions for the various methods.

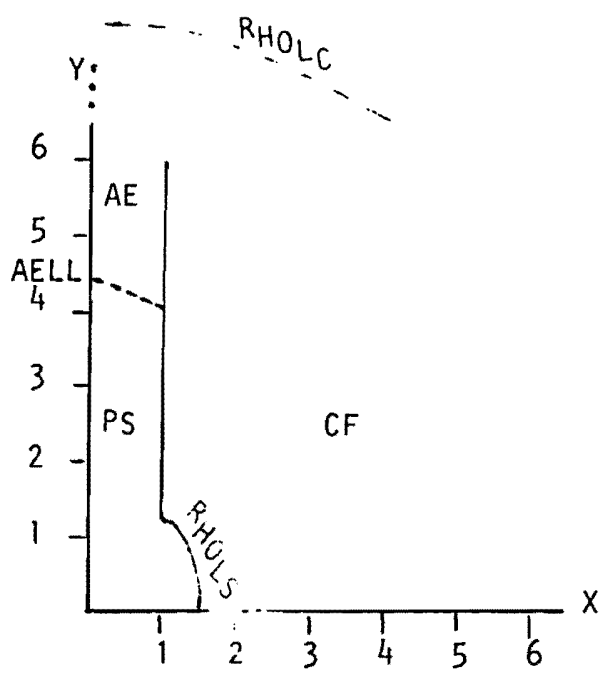

Figure 1. Parameter plane.

The dividing line RHO = AELL between the use of the power series and the asymptotic expansion and RHO $=$ RHOLC are the only boundaries subject to the required precision. Single and double precision results, for example on the Univac 1108, are the results of two different methods in the region where RHO roughly lies between 4.3 and 6.4 .

This mapping of the region ensures for the required precision that the least number of terms are computed and the loss of significance is kept to a minimum. While the second form of the power series is preferable for real positive $z$, since all terms are positive, the first form ensures greater accuracy for complex $z$ since the real and imaginary parts of the terms may be positive, negative or zero for any $\mathrm{RN}(=n)$. The power series is there more rapidly convergent; the relative error may then be approximated by the ratio of the term to the sum of terms; comparison of this approximant with the tolerance for the relative error controls the number of terms needed. Since the terms tend to zero through underflow, there will always be convergence even if the tolerance is made considerably smaller than the precision of the machine. The power series is evaluated using recurrence relations in the following form:

$$
\text { ERFZ }=\frac{\text { TWO }}{\text { SQRT(PI) }} \underset{\text { RN }=0}{\mathrm{RNF}} \mathrm{SGN}(\mathrm{RN})^{*} \mathrm{TM}(\mathrm{RN})=\frac{\mathrm{TWO}}{\mathrm{RTPI}} \cdot \mathrm{SUM}
$$

where

$$
\begin{aligned}
& \mathrm{SGN}(0)=1, \mathrm{SGN}(\mathrm{RN}+1)=-\mathrm{SGN}(\mathrm{RN}) \\
& \mathrm{TM}(\mathrm{RN})=\left(\left(\mathrm{AZ} * *\left(2^{*} \mathrm{RN}+1\right)\right) / 1 * 2^{*} \ldots \mathrm{RN}\right) /(2 * \mathrm{RN}+1) \\
& \mathrm{TM}(\mathrm{RN})=\mathrm{PTM}(\mathrm{RN}) / \mathrm{DN}(\mathrm{RN}) \\
& \mathrm{PTM}(0)=\mathrm{AZ}, \mathrm{PTM}(\mathrm{RN}+1)=(\mathrm{AZ} * * 2)^{*} \mathrm{PTM}(\mathrm{RN}) /(\mathrm{RN}+1) \\
& \mathrm{DN}(0)=1, \mathrm{DN}(\mathrm{RN}+1)=\mathrm{DN}(\mathrm{RN})+2
\end{aligned}
$$


In determining the terminal value of $\mathrm{RN}$, normalization is necessary to avoid overflows and underflows. The normalization factor TMAX is the maximum of the absolute value of the real and imaginary parts of TM and SUM. If TMAX equals zero or $\mid$ TM/TMAX $\left.\right|^{2}$ underflows, RNF $=$ RN.

If $|\mathrm{SUM} / \mathrm{TMAX}|^{2}$ underflows, additional terms are obtained. Otherwise, if |TM/TMAX $\left.\right|^{2 / \mid S U M /}$ TMAX $\left.\right|^{2}$ is less than TOLER ${ }^{2}$, then RNF $=$ RN.

The continued fraction expansion starts to converge more slowly as $z$ tends to zero. The "even" form is used since the required number of terms is halved at the expense of very little extra computation for successive numerators and denominators. The continued fraction is evaluated by using the recurrence relations in the "forward" direction. The number of terms needed is determined by checking to see if the relative error of two successive convergents is less than the tolerance. On the other hand, if the relative error remains constant or starts to increase, the recurrence is terminated and the prior convergent taken as the value of the continued fraction. In this way, the process is always terminated when maximum precision is attained.

The "even" form of the continued fraction takes on the following implementation:

$$
\mathrm{EZ2CZ}=\frac{2^{*} \mathrm{AZ}}{\mathrm{RTPI}} \stackrel{\mathrm{RNF}}{\mathrm{RN}}=1 \frac{\mathrm{AM}(\mathrm{RN})}{\mathrm{BM}(\mathrm{RN})}
$$

with

$$
\operatorname{AM}(1)=1, \operatorname{AM}(\mathrm{RN}+1)=-\mathrm{WM}(\mathrm{RN}+1)^{*}(\mathrm{WM}(\mathrm{RN}+1)+1)
$$$$
\mathrm{BM}(1)=2^{*}(\mathrm{AZ} * 2)+1, \mathrm{BM}(\mathrm{RN}+1)=\mathrm{BM}(\mathrm{RN})+4
$$

where

$$
\begin{aligned}
& \mathrm{WM}(1)=-1, \mathrm{WM}(\mathrm{RN}+1)=\mathrm{WM}(\mathrm{RN})+2 \\
& \mathrm{EZ2CZ}=\left(\mathrm{AZ}{ }^{*}(\mathrm{FM} / \mathrm{GM})\right)^{*} 2 / \mathrm{RTPI}=(\mathrm{AZ} * \mathrm{~F}(\mathrm{RN}))^{*} 2 / \mathrm{RTPI}
\end{aligned}
$$

where

$$
\begin{aligned}
& \operatorname{FM}(-1)=1, \operatorname{FM}(0)=0 \\
& \operatorname{GM}(-1)=0, \operatorname{GM}(0)=1
\end{aligned}
$$

and

$$
\begin{aligned}
& \mathrm{FM}(\mathrm{RN})=\mathrm{BM}(\mathrm{RN}) * \mathrm{FM}(\mathrm{RN}-1)+\mathrm{AM}(\mathrm{RN}) * \mathrm{FM}(\mathrm{RN}-2) \\
& \mathrm{GM}(\mathrm{RN})=\mathrm{BM}(\mathrm{RN})^{*} \mathrm{GM}(\mathrm{RN}-1)+\mathrm{AM}(\mathrm{RN})^{*} \mathrm{GM}(\mathrm{RN}-2)
\end{aligned}
$$

The relative error may be approximated by $[F(R N)-F(R N-1)] / F(R N)=R E(R N)$. If the modulus squared of the relative error REM2(RN) is less than the square of the tolerance divided by $8, R N F=R N$. If REM2(RN) is greater than or equal to REM2(RN - 1), then RNF = RN - 1. Normalization is likewise necessary here to avoid overflows in computing the relative error and its modulus squared and also in the generation of the successive convergents.

The asymptotic expansion is likewise evaluated using recurrence relations in the following form:

$$
\mathrm{EZ2CZ}=\frac{1}{\mathrm{SQRT}(\mathrm{PI})} \sum_{\mathrm{RN}=0}^{\mathrm{RNF}} \mathrm{SGN}(\mathrm{RN})^{*} \mathrm{TM}(\mathrm{RN})=\mathrm{SUM} / \mathrm{RTPI}
$$

where

$$
\begin{aligned}
\operatorname{SGN}(0) & =1, \mathrm{SGN}(\mathrm{RN}+1)=-\mathrm{SGN}(\mathrm{RN}) \\
\mathrm{TM}(\mathrm{RN}) & =(1 / \mathrm{AZ})^{*}\left(1^{*} 3^{*} \ldots\left(2^{*} \mathrm{RN}-1\right)\right) /\left(2 *\left(\mathrm{AZ}{ }^{* *} 2\right)\right)^{* *} \mathrm{RN} \\
\mathrm{TM}(0) & =1 / \mathrm{AZ}, \mathrm{TM}(\mathrm{RN}+1)=\mathrm{DN}(\mathrm{RN}+1)^{*} \mathrm{TM}(\mathrm{RN}) *\left(1 /\left(2^{*}\left(\mathrm{AZ}^{* *} 2\right)\right)\right)
\end{aligned}
$$

with

$$
\mathrm{DN}(1)=1, \mathrm{DN}(\mathrm{RN}+1)=\mathrm{DN}(\mathrm{RN})+2 .
$$


The relative error may be approximated here by the ratio TM/SUM. The convergence test precedes the divergence test and is implemented as REM2 less than (TOLER**2)/8 to attain greater accuracy in both the real and imaginary parts. If the modulus squared of the term remains the same or increases, the prior sum is taken as the final sum.

For $z$ along the imaginary axis, the error function is purely imaginary; the real part of erfc $z=1$ and of $e^{z^{2}} \operatorname{erfc} z=e^{-(A Z 1)^{2}}$. No difficulties arise in the use of the power series. However, since the asymptotic expansion is given for $|z| \rightarrow \infty$, the correction must be applied for AZR $\rightarrow 0$.

The following table gives an indication of the number of terms needed to obtain maximum machine accuracy on the Univac 1108 with the various methods of computation.

\begin{tabular}{lcc}
\hline Method & \multicolumn{2}{c}{ Number of Terms } \\
\hline & Single Precision & Double Precision \\
& TOLER $=.745 \mathrm{E}-8$ & TOLER $=.867 \mathrm{D} \cdot 18$ \\
\hline Power Series & 50 & 112 \\
Continued Fraction & 25 & 99 \\
Asymptotic Expansion & 22 & 45 \\
\hline
\end{tabular}

\section{Range}

If the real part of $z$ is zero or positive, $e^{z^{2}}$ erfc $z$ is valid for $z$ throughout the entire machine range. Otherwise, the real part of $z^{2}$ is essentially limited by the range of the exponential library subroutine with the imaginary part of $z^{2}$ limited by the range of the sine, cosine library subroutine.

\section{Accuracy and Precision}

The maximum relative error, generally in erfc $z$, except for regions in the immediate neighborhood of zeros of the real and/or imaginary parts of the functions is $8 \mathrm{E}-6$ for single precision computation on the Univac 1108.

The precision may be varied by changing the value of TOLER.

\section{Timing (Univac 1108 Time/Sharing Executive System)}

The time estimates given below are highly dependent on the operating system environment and consequently should not be relied on for critical timing measurements.

\begin{tabular}{|c|c|c|}
\hline \multirow{5}{*}{$\begin{array}{l}\text { Region } \\
\mathrm{ZR}=0(.1) 4, \mathrm{ZI}=0(.2) 8 \\
\quad \text { Nethod }\end{array}$} & \multicolumn{2}{|c|}{ Time (Seconds) } \\
\hline & \multirow{3}{*}{$\begin{array}{c}\text { Single Precision } \\
\text { TOLER }=.745 E-8 \\
5.94\end{array}$} & \multirow{3}{*}{$\begin{array}{c}\text { Double Precision } \\
\text { TOLER }=.867 \mathrm{D} \cdot 18 \\
22.5\end{array}$} \\
\hline & & \\
\hline & & \\
\hline & $M$ & ion \\
\hline Power Series & .0101 & .038 \\
\hline Continued Fraction & .0088 & .052 \\
\hline Asymptotic Expansion & .0035 & .0093 \\
\hline
\end{tabular}

\section{Testing}

The language of the subroutine was checked for conformity with the PFORT VERIFIER. ${ }^{3}$ Test arguments were devised and used in the analysis of the subroutine with the PROFILER. ${ }^{4}$

\footnotetext{
-The PFORT Verifier, A. D. Hall and B. G. Ryder, Bell Laboratories, Murray Hill, N.J. Proceedings of the Computer Science and Statistics Eighth Annual Symposium on the Interface, University of California, Los Angeles, February 13-14, 1975.

- Program Execution Profles, G. Sande, World Bank, Washington, D.C. Proceedings of the Computer Science and Statistics Eighth Annual Symposium on the Interface, University of California, Los Angeles, February 13-14, 1975.
} 
The subroutine was used to obtain related functions which were checked against available published tables ([1]-[5], [7]-[9], [13], [15]). Single precision results covering the $9 \times 9$ grid were compared against double precision results. This precision test particularly verified that the scaling operations were valid and undetected overflows had not occurred.

Where applicable, results were obtained by independent methods, for example, the power series and continued fraction, the power series and asymptotic expansion or all three methods. Otherwise, both forms of the power series or continued fraction were used. In addition, numerical integration of various forms of the integral representation was employed.

\section{Test Values}

Six-significant figure tables of erfe $z, e^{x^{2}}$ erfc $z$ and $e^{z^{2}} \operatorname{erfc}(-z)$ are included in the appendix. The computations were performed with double precision arithmetic to provide more accurate values for checking purposes. The arguments are in polar coordinate form $z=\varrho e^{i \theta}$ for $\varrho=0(.02) .2(.1) 3(.5) 9, \Theta=0^{\circ}\left(15^{\circ}\right) 30^{\circ}$, $37.5^{\circ}, 45^{\circ}, 50^{\circ}\left(10^{\circ}\right) 90^{\circ}$. Values of the functions for $z$ in other quadrants are readily obtainable with symmetry relations.

\section{Argand Diagram of erf $x=\frac{2}{\sqrt{ } \pi} \int_{0}^{z=\rho e^{i \theta}} e^{-t^{2}} d t$}

Since erf $z$ and erfe $z$ are complementary functions, erf $z$ is not tabulated but the argand diagram of the function is included to illustrate divergent and convergent spirals.

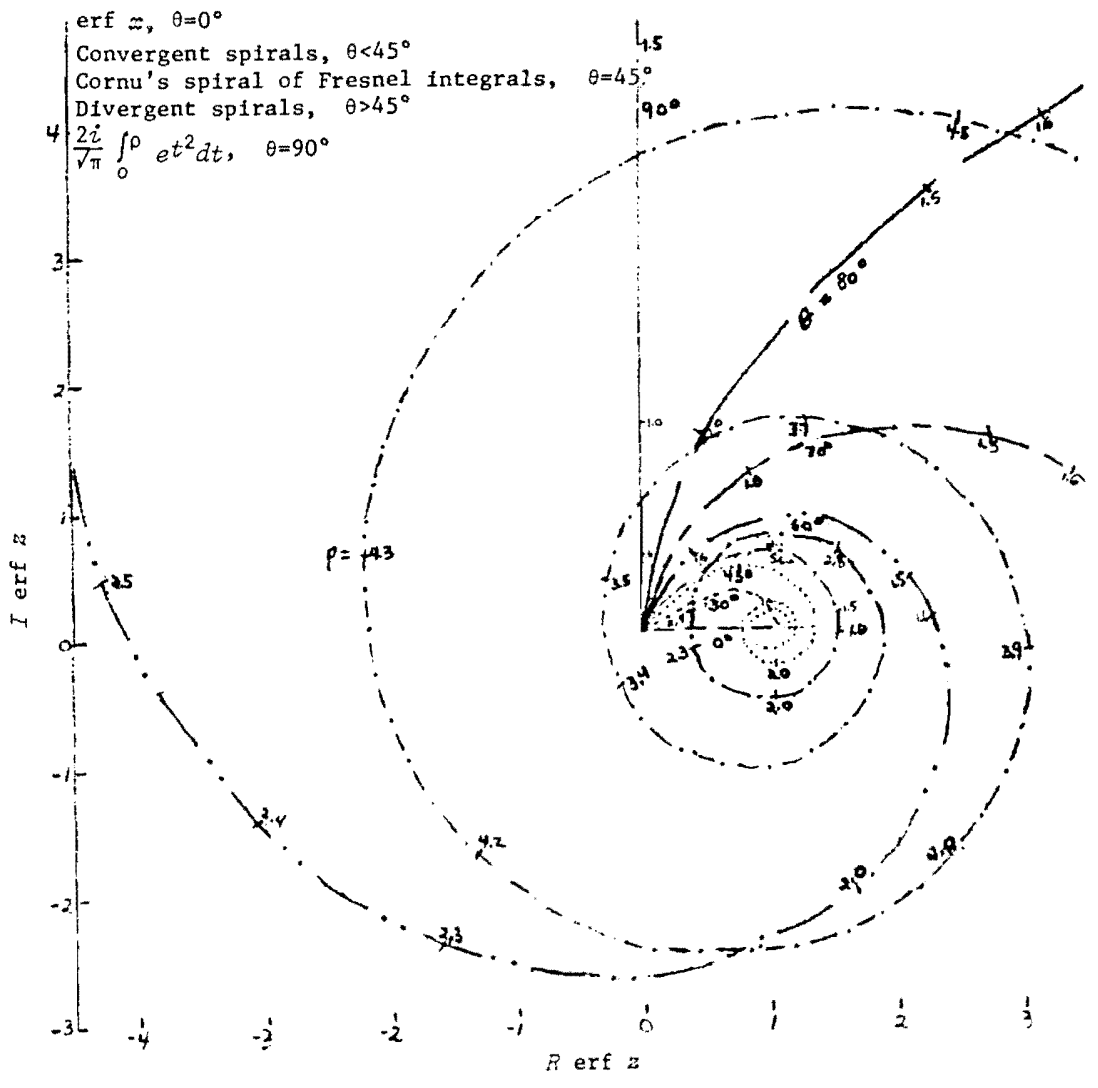




\section{Special Values}

Relevant values are collected here for completeness and ease of modification and checking of the program.

Zeros

$$
\text { erf } z_{s}=0
$$

$\begin{array}{lc}s & z_{s} \\ 1 & 1.45061616+\mathrm{i} 1.88094300 \\ 2 & 2.24465927+\mathrm{i} 2.61657514 \\ 3 & 2.83974105+\mathrm{i} 3.17562810\end{array}$

$$
\begin{array}{cc} 
& w\left(z_{z}\right)=0 \\
s & z_{z} \\
1 & 1.99146684-\mathrm{i} 1.35481013 \\
2 & 2.69114902-\mathrm{i} 2.17704491 \\
3 & 3.23533087-\mathrm{i} 2.78438761
\end{array}
$$

$\begin{array}{ccc} & C\left(z_{s}\right)=0 & S\left(z_{s}\right)=0 \\ s & z_{s} & z_{s} \\ 1 & 1.7437+\mathrm{i} .3057 & 2.0093+\mathrm{i} .2885 \\ 2 & 2.6515+\mathrm{i} .2529 & 2.8335+\mathrm{i} .2443 \\ 3 & 3.3204+\mathrm{i} .2240 & 3.4675+\mathrm{i} .2185\end{array}$

Maxima and Minima of Fresnel Integrals

$\begin{array}{lllll}s & M_{s}=C(\sqrt{4 s+1}) & m_{s}=C(\sqrt{4 s+3}) & M_{s}^{*}=S(\sqrt{4 s+2}) & m_{s}^{*}=S(\sqrt{4 s+4}) \\ 0 & .779893 & .321056 & .713972 & .343416 \\ 1 & .640807 & .380391 & .628940 & .387969 \\ 2 & .605721 & .404261 & .600362 & .408301 \\ 3 & .588128 & .417922 & .584942 & .420516\end{array}$

Radius of Univalence $\varrho$

$\begin{array}{ll}\int_{0}^{x} e^{-r^{2}} d t & e^{x^{2}} \int_{0}^{x} e^{-r^{2}} d t \\ \varrho=1.5748376 & .92413887\end{array}$

Maximum and Inflection Point for Dawson's Integral

$F(.9241388730)=.5410442246$

$F(1.5019752682)=.4276866160$ 


$$
\begin{aligned}
& 1^{\circ}=1.74532925199432957692369076848861271(-2) r \\
& \pi=3.14159265358979323846 \quad 26433 \quad 8327950288 \\
& \pi / 2=1.570796326794896 \quad 61923 \quad 13216 \quad 91639 \quad 75144 \\
& \sqrt{ } \pi=1.77245 \quad 3850905516 \quad 02729 \quad 81674 \quad 83341 \quad 14518 \\
& \sqrt{ } \pi / 2=.88622 \quad 692545275801364908374167057259 \\
& 2 \pi=6.28318 \quad 53071 \quad 79586 \quad 47692 \quad 52867 \quad 66559 \quad 00576 \\
& 2 \sqrt{ } \pi=3.5449077018 \quad 11032054596334966682 \quad 29036 \\
& \mathrm{e}=2.71828 \quad 18284 \quad 59045 \quad 23536 \quad 02874 \quad 71352 \quad 66249 \\
& 1 / \pi=.31830 \quad 98861 \quad 83790 \quad 67153 \quad 77675 \quad 26745 \quad 02872 \\
& 2 / \pi=.63661 \quad 9772367581 \quad 34307 \quad 5535053490 \quad 05744 \\
& 1 / \sqrt{ } \pi=.564189583547756 \quad 28694 \quad 80794 \quad 5156077258 \\
& 2 / \sqrt{ } \pi=1.12837 \quad 91670 \quad 95512 \quad 5738961589 \quad 03121 \quad 54517 \\
& 1 / 2 \pi=.15915494309189533576888376337251436 \\
& 1 / 2 \sqrt{ } \pi=.28209479177387814347403972578038629 \\
& 1 / \mathrm{e}=.36787944117144232159552377016146086 \\
& \text { erf } 1=.84270 \quad 079294971486934 \quad 12206 \quad 3508260926 \\
& \int_{0}^{1} e^{-r^{2}} d t=(\sqrt{ } \pi / 2) \operatorname{erf} 1=\sum_{n=0}^{\infty} \frac{(-1)^{n}}{n !(2 n+1)}=.74682413281242702539946743613185300 \\
& (\sqrt{ } \pi / 2) e^{1} \operatorname{erf} 1=\sum_{n=0}^{\infty} \frac{2^{n}}{1 \cdot 3 .(2 n+1)}=2.03007846927870497553908992566595044 \\
& \int_{0}^{1} e^{t^{2}} d t=\sum_{n=0}^{\infty} \frac{1}{n !(2 n+1)}=1.46265174590718160880404858685698815 \\
& \sum_{n=0}^{\infty} \frac{1}{(2 n) !(4 n+1)}=1.10473 \quad 79393 \quad 59804 \quad 31710 \quad 17580 \quad 11494 \quad 42058 \\
& \sum_{n=0}^{\infty} \frac{1}{(2 n+1) !(4 n+3)}=.35791380654737729170229057536256757 \\
& \sum_{n=0}^{\infty} \frac{2^{2 n}}{1 \cdot 3 \ldots(4 n+1)}=1.28407898809573669733773867303675360 \\
& \sum_{n=0}^{\infty} \frac{2^{2 n+1}}{1 \cdot 3 \ldots(4 n+3)}=.74599948118296827820135125262919684 \\
& e^{-1} \int_{0}^{1} e^{t^{2}} d t=\sum_{n=0}^{\infty} \frac{(-1)^{n} 2^{n}}{1 \cdot 3 . .(2 n+1)}=.53807950691276841913638742040755675
\end{aligned}
$$

Typical Tolerances and Their Natural Logarithms

$$
\begin{aligned}
& 2^{-16}=0.152587890625(-4) \\
& 2^{-24}=.59604644775390625(-7) \\
& 2^{-27}=.7450580596923828125(-8) \\
& 2^{-36}=.14551915228366851806640625(-10) \\
& 2^{-48}=.3552713678800500929355621337890625(-14) \\
& 2^{-56}=.1387778780781445675529539585113525390625(-16) \\
& 2^{-60}=\quad .867361737988403547205962240695953369140625(-18) \\
& 2^{-108}=\quad .3081487911019577364889564708135883709660962637144621112 \\
& 383902072906494 \quad 140625(-32)
\end{aligned}
$$

$\log _{.}\left(2^{-16}\right)=-11.09035488895912495067571394333082508$

$\log _{.}\left(2^{-24}\right)=-16.63553233343868742601357091499623763$

$\log _{.}\left(2^{-27}\right)=-18.71497387511852335426526727937076733$ 


$$
\begin{array}{rlllllll}
\log _{e}\left(2^{-36}\right) & =-24.95329 & 85001 & 58031 & 13902 & 03563 & 72494 & 35645 \\
\log _{e}\left(2^{-48}\right) & =-33.27106 & 46668 & 77374 & 85202 & 71418 & 29992 & 47526 \\
\log _{e}\left(2^{-56}\right) & =-38.81624 & 21113 & 56937 & 32736 & 49988 & 01657 & 88781 \\
\log _{e}\left(2^{-60}\right) & =-41.58883 & 08335 & 96718 & 56503 & 39272 & 87490 & 59408 \\
\log _{e}\left(2^{-108}\right) & =-74.85989 & 55004 & 74093 & 41706 & 10691 & 17483 & 06935
\end{array}
$$

Maximum and Minimum Machine Values and Their Natural Logarithms (Univac 1108 Single and Double Precision Limits)

$\mathrm{NBC}=$ Number of binary digits in the (biased) characteristic of a floating point number

$$
\begin{aligned}
& 2^{-\left(2^{N B C-1}+1\right)} \leq \mathrm{x}<2^{2^{N B C-1}-1} \\
& \mathrm{NBC}=8
\end{aligned}
$$

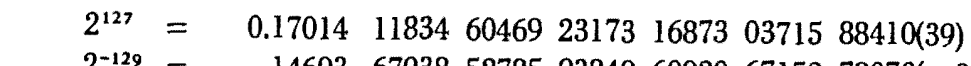

$$
\begin{aligned}
& 2^{-129}=.14693679385278593849609206715278070(-38) \\
& \log _{e}\left(2^{127}\right)=88.02969193111305429598847942518842414 \\
& \log _{e}\left(2^{-129}\right)=-89.41598629223294491482294366810477728 \\
& \mathrm{NBC}=11 \\
& 2^{1023}=0.89884656743115795386465259539451236(308) \\
& 2^{-1025}=.27813423231340017288627908966655050(-308) \\
& \log _{e}\left(2^{1023}\right)=709.08956571282405153382846025171462914 \\
& \log _{e}\left(2^{-1025}\right)=-710.47586007394394215266292449463098227
\end{aligned}
$$

\section{References}

[1] Abramowitz, M. and Stegun, I. A. Handbook of Mathematical Functions. Nat. Bur. Stand. (U.S.) Appl. Math. Ser. 55; 1964.

[2] Faddeeva, V. N. and Terent'ev, N. M. Tables of Values of the Function $w(z)=e^{-z^{2}}\left[1+(2 i \sqrt{ } \pi) \int_{0}^{3} e^{2^{2}} d t\right]$ for Complex Arguments. New York: English Trans., Pergamon Press; 1961.

[3] Fettis, H. E., Caslin J. C. and Cramer, K. R. Complex Zeros of the Error Function and of the Complementary Error Function. Math. Comp. 27, 122: 401-407; 1973.

[4] Finn, G. D. and Mugglestone, D. Tables of the Line Broadening Function H(a,v). Mon. Not. R. Astr. Soc. 129: 221-235; 1965.

[5] Fried, B. D. and Conte, S. D. The Plasma Dispersion Function, The Hilbert Transform of the Gaussian. New York: Academic Press; 1961.

[6] Gautschi, W. Efficient Computation of the Complex Error Function. SIAM J. Numer. Anal. 7, 1: 187-198; 1970.

[7] Hummer, D. C. The Voigt Function: An Eight-Significant Figure Table and Generating Procedure. Mem. Roy. Astronom, Soc. 70: $1-31,1965$.

[8] Karpov, K. A. Tablitsy Funktsii $w(z)=e^{-x^{2}} \int_{0}^{x} e^{x^{2}} d x$ v Kompleksnoi Oblasti. Moscow, U.S.S.R.: Izdat. Akad. Nauk S.S.S.R.; 1954.

[9] Karpov, K.A. Tablitsy Funktsii $F(z)=\int_{0}^{x} e^{x^{2}} d x$ v Kompleksnoi Oblasti. Moscow, U.S.S.R.: Izdat. Akad. Nauk S.S.S.R.; 1958.

[10] Kreyszig, E. and Todd, J. The Radius of Univalence of the Error Function. Numerische Mathematik $1: 78-89 ; 1959$.

[11] Kreyszig, E. and Todd, J. On the Radius of Univalence of the Function $\exp z^{2} \int_{0}^{x} \exp \left(-t^{2}\right) d t$. Pacific Journal of Mathematics 9 , 1: $123-127 ; 1959$.

[12] Lohmander, B. and Rittsten, S. Table of the Function $y=e^{-x^{2}} \int_{0}^{x} e^{t^{2}} d t$. Kungl. Fysiogr. Sallsk. i Lund Forh. 28: 45-52; 1958.

[13] Martz, C. W. Tables of the Complex Fresnel Integral. NASA Report SP-3010; Washington; 1964.

[14] Pearcey, T. Table of the Fresnel Integral. London, England: Cambridge University Press; 1956.

[15] Rosser, J. B. Theory and Application of $\int_{0}^{x} e^{-x^{2}} d x$ and $\int_{0}^{x} e^{-p^{2} y^{2}} d y \int_{0}^{x} e^{-x^{2}} d x$. Brooklyn, NY: Mapleton House; 1948.

[16] Tablitsy Integralov Frenelya. Moscow, U.S.S.R.: Izdat. Akad. Nauk S.S.S.R.; 1953.

[17] Van Wijngaarden, A. and Scheen, W. L., Table of Fresnel Integrals. Verh Nederl. Akad. Wetensch. Afd. Natuurk. Sec. I, 19, 4: 1-26; 1949. 


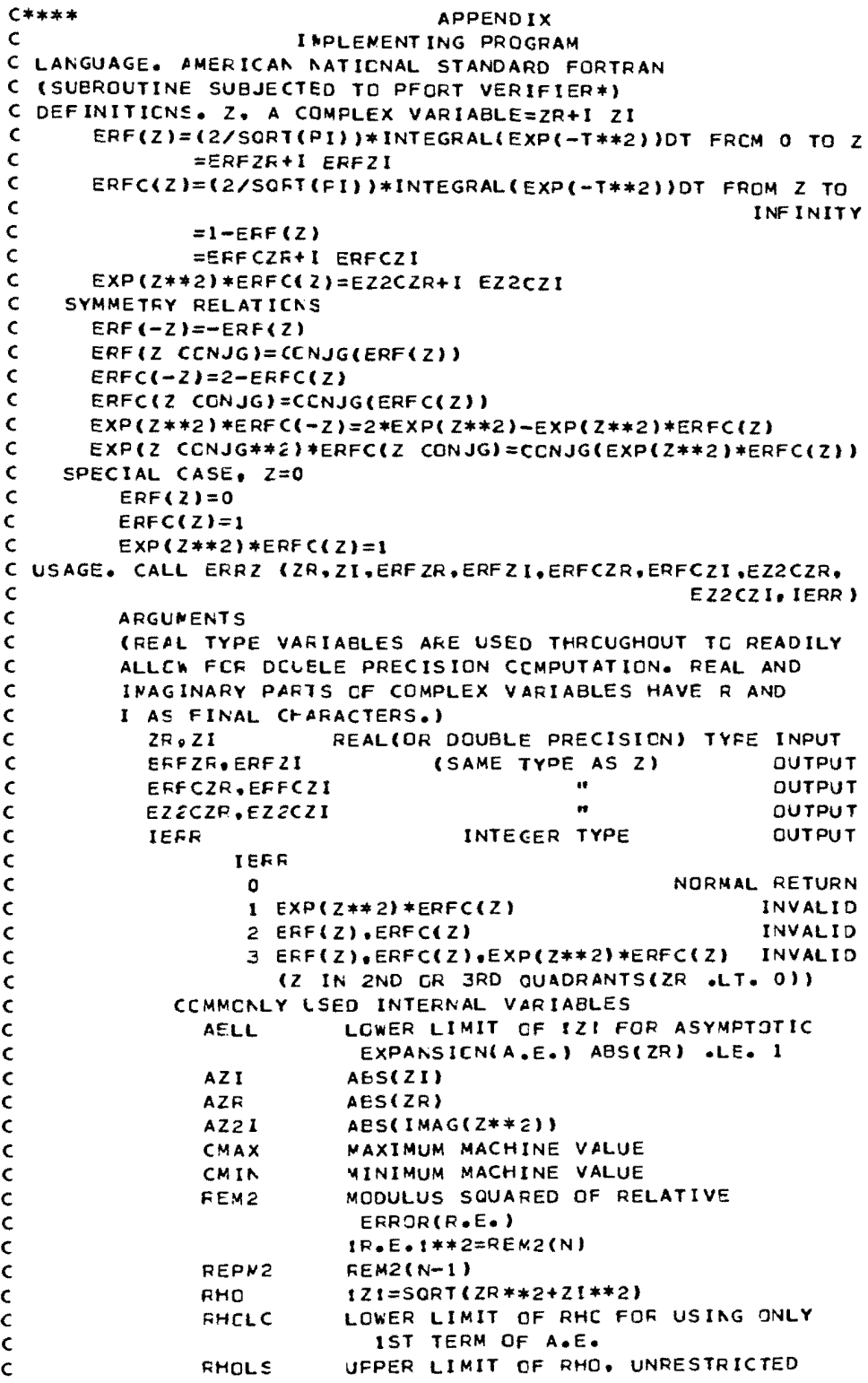




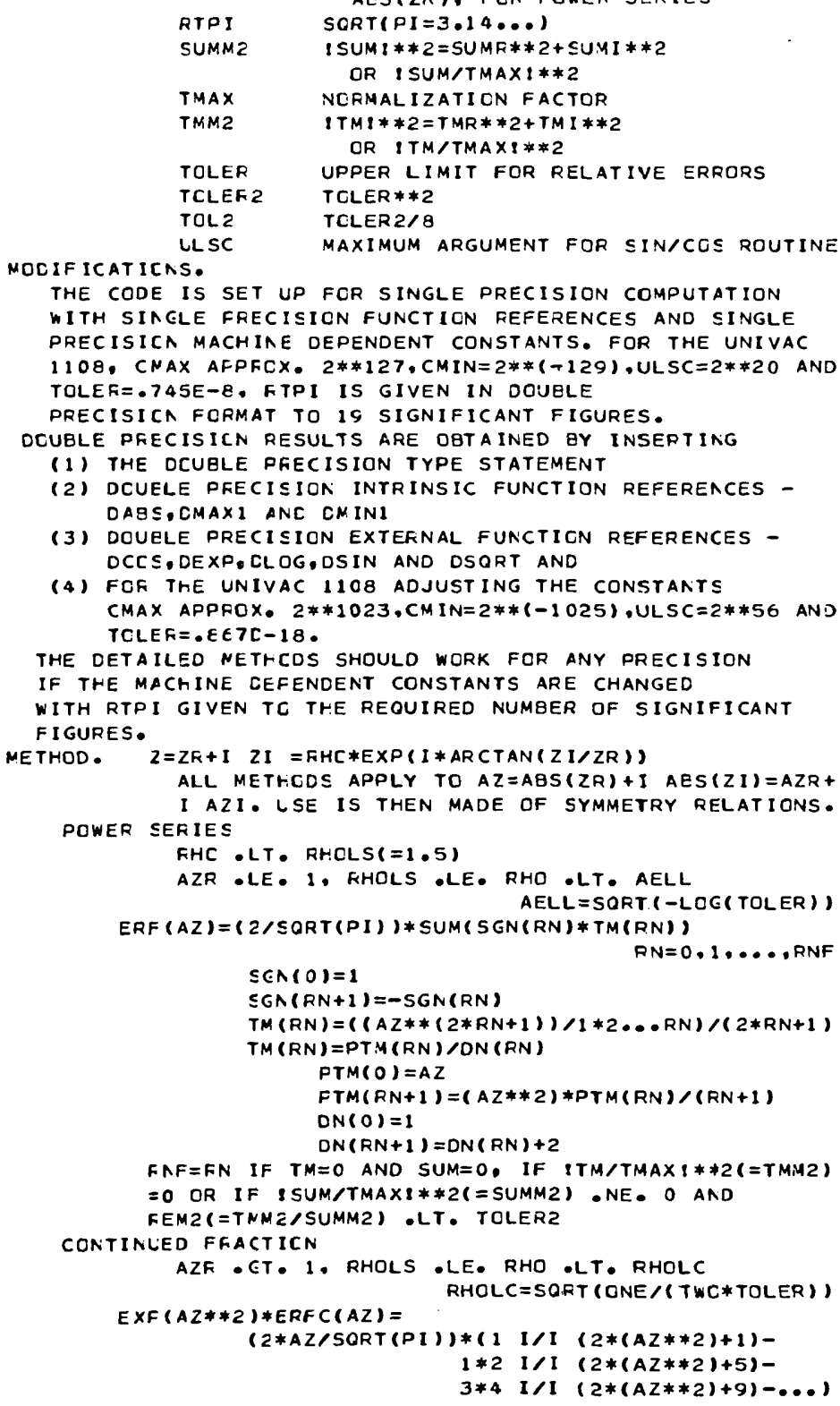




\begin{tabular}{|c|c|c|}
\hline $11 t$ & $c$ & $=(Z \# A Z / F T P I) * I I(A M(R N) I / I E N(R N))$ \\
\hline 117 & $c$ & $R N=1,2, \ldots . R N F$ \\
\hline $11 \%$ & $c$ & $A N(1)=1$ \\
\hline $11 \varsigma$ & $c$ & $A N(A N+1)=-K H(R N+1) *(W N(R N+1)+1)$ \\
\hline 120 & $c$ & $\operatorname{FN}(1)=2 *(A Z * * 2)+1$ \\
\hline $1=1$ & c & $B M(R N+1)=\operatorname{EN}(R A)+4$ \\
\hline $12 \bar{z}$ & c & WM $(1)=-1$ \\
\hline $1 \approx z$ & $c$ & $\triangle M(F N+1)=W M(F N)+2$ \\
\hline 124 & $c$ & $=(A Z *(F M / G M)) * 2 / R T F I$ \\
\hline $1<5$ & c & $=(A Z * F(F N)) * 2 / F T P I$ \\
\hline 126 & $C$ & $f N(-1)=1$ \\
\hline $1 \tilde{2} 7$ & $c$ & $6 N(-1)=0$ \\
\hline $1 \approx F$ & $c$ & $F N(a)=0$ \\
\hline $1 \ddot{2}$ & $c$ & $G N(0)=1$ \\
\hline $1 \equiv 0$ & C & $F N(K N)=B M(K N) * F N(R N-1)+A M(R N) * F N(R N-2)$ \\
\hline $1 \equiv 1$ & $C$ & $G N(F N)=B M(R N) * G M(F N-1)+A M(R N) * C N(R N-2)$ \\
\hline $1 \equiv \tilde{z}$ & $C$ & $F N F=F A$ IF $F E M Z\left(F C R R_{\bullet} E_{\bullet}=(F(F N)-F(F N-1)) / F(R N)\right)$ \\
\hline $1 \Xi \Xi$ & C & -LT. ICLZ OR \\
\hline 134 & c & FNF $=F A-1$ IF REM2(RN) E GE. FEMZ(FN-I) \\
\hline $13 \leq$ & c & ASYNFTCTIC EXFAASICN \\
\hline $1 \Xi c$ & C & AZF -LE. 1, AELL .LE. FrC .LT, FHOLC \\
\hline $1 \geq 7$ & c & IFCF FHCLC ILE. RHC -LE. CNAX, TO PFEsefive \\
\hline $1 \equiv 3$ & $c$ & ACCUFACY AN ALTERNATIVE CCMPUTATICA OF \\
\hline $1 \geq 9$ & c & THE FIFST TERN CF THE A.E. IS EMFLCYED.) \\
\hline $14 \mathrm{C}$ & $c$ & $E X F(A Z * * Z) * E F F(A Z)=(\operatorname{SUM}(S \in N(F N) * T M(K N))) / S O R T(P I)$ \\
\hline 141 & $c$ & $R N=0,1, \ldots$ RNF \\
\hline $14 \bar{z}$ & $c$ & $S G N(0)=1$ \\
\hline 143 & c & $\operatorname{SEA}(F A+1)=-\operatorname{SGN}(R N)$ \\
\hline 144 & c & $T N(F N)=(1 / A L) *(1 * 3 \ldots(2 * R N-1)) /$ \\
\hline 145 & $c$ & $(2 *(A Z * * 2)) * * R N$ \\
\hline $14 \epsilon$ & c & $T M(C)=1 / A Z$ \\
\hline 147 & $C$ & $T N(F A+1)=C N(K N+1) *(T M(R N) *(1 /(2 *(A Z * * 2)))$ \\
\hline 148 & $\mathrm{c}$ & $c \wedge(1)=1$ \\
\hline 145 & $c$ & $C N(F N+1)=D N(F N)+2$ \\
\hline 150 & c & $F \cap F=F \wedge \quad I F \quad F E M 2\left(F C R \quad F \cdot E_{0}=T N / S U M\right) \cdot L T \cdot T O L 2$ \\
\hline $1 \leqslant 1$ & $c$ & FAF $=F A-1$ IF TMMZ(FN) $\cdot$ GE. TMPNZ $(=T M M Z(R N-1))$ \\
\hline $1 \subseteq 2$ & $c$ & (OIVERGENCE) \\
\hline $1 \leqslant 3$ & $c$ & RAAEE. \\
\hline $1 \subseteq 4$ & C & $\operatorname{EXP}(Z * * Z) * E F F(2)$ IS VALTO FOR $Z R$. GE. O THECLGMOUT \\
\hline $1 \leqslant 5$ & $\mathbf{c}$ & THE EAITIZE NACHIAE RANGE, ERF $(Z), E R F C(Z)$ ANC \\
\hline $1 \leqslant \epsilon$ & $c$ & EXF $(2 * * 2) * E F F C(2)(F C R$ ZR .LT. C) ARE LIMITED EY THE \\
\hline $1 \subseteq 7$ & $\mathrm{C}$ & FARGE AND ACCLFACY OF TRE SINE.COSINE AND/OA THE \\
\hline $15 \varepsilon$ & c & EXFCMENTIAL LIEFAFYY ROUTINES. \\
\hline 155 & $c$ & ACCURACY. THE NAXINUN FELATIVE ERRCF (GENERALLY IN ERFC) \\
\hline 160 & c & EXCEPT IN THE IMMEDIATE NEIGHECAMCCD CF ZERCS, \\
\hline $1 \in 1$ & C & IS $E(-E)$ CN THE UNIVAC $110 E$ FCR \\
\hline $1 \in 2$ & c & SINGLE FFEGISICN CCMPUTATICN, THE REAL \\
\hline $1 \in \Xi$ & c & ANE INAEINAFY PARTS INDEPENDENTLY AS WELL \\
\hline $1 \in 4$ & $c$ & AS THEIF ZEFCS ENTER INTC CONSIDERATICN. \\
\hline 165 & c & PRECISICN. VARIAELE - EY SETTING A PREDETERNINEC VALUE OF \\
\hline $1 \epsilon \epsilon$ & c & TCLER \\
\hline $1 \in 7$ & c & UNIVAC $110 E$ TIME/SHARING EXECUTIVE SYSTEM \\
\hline 168 & $c$ & TINING. \\
\hline 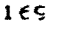 & c & (SECCNCS) \\
\hline 170 & c & STCRAGE. 1171 WCFCS RECUIFED EY THE UNIVAC 1108 CCMPILER \\
\hline 71 & c & (I13 FCFTFAN STATEMENTS, 95 VARIABLES \\
\hline $\begin{array}{l}17 \bar{z} \\
17 \Sigma\end{array}$ & $c$ & $\begin{array}{l}\text { THE PFCFT VEFIFIEF,A.C.HALL AND B.C.RYDER } \\
\text { GEELL LAECFATCRIES, NURRAY HILL. N.J.J PRCC.CF THE COMPUTE }\end{array}$ \\
\hline
\end{tabular}




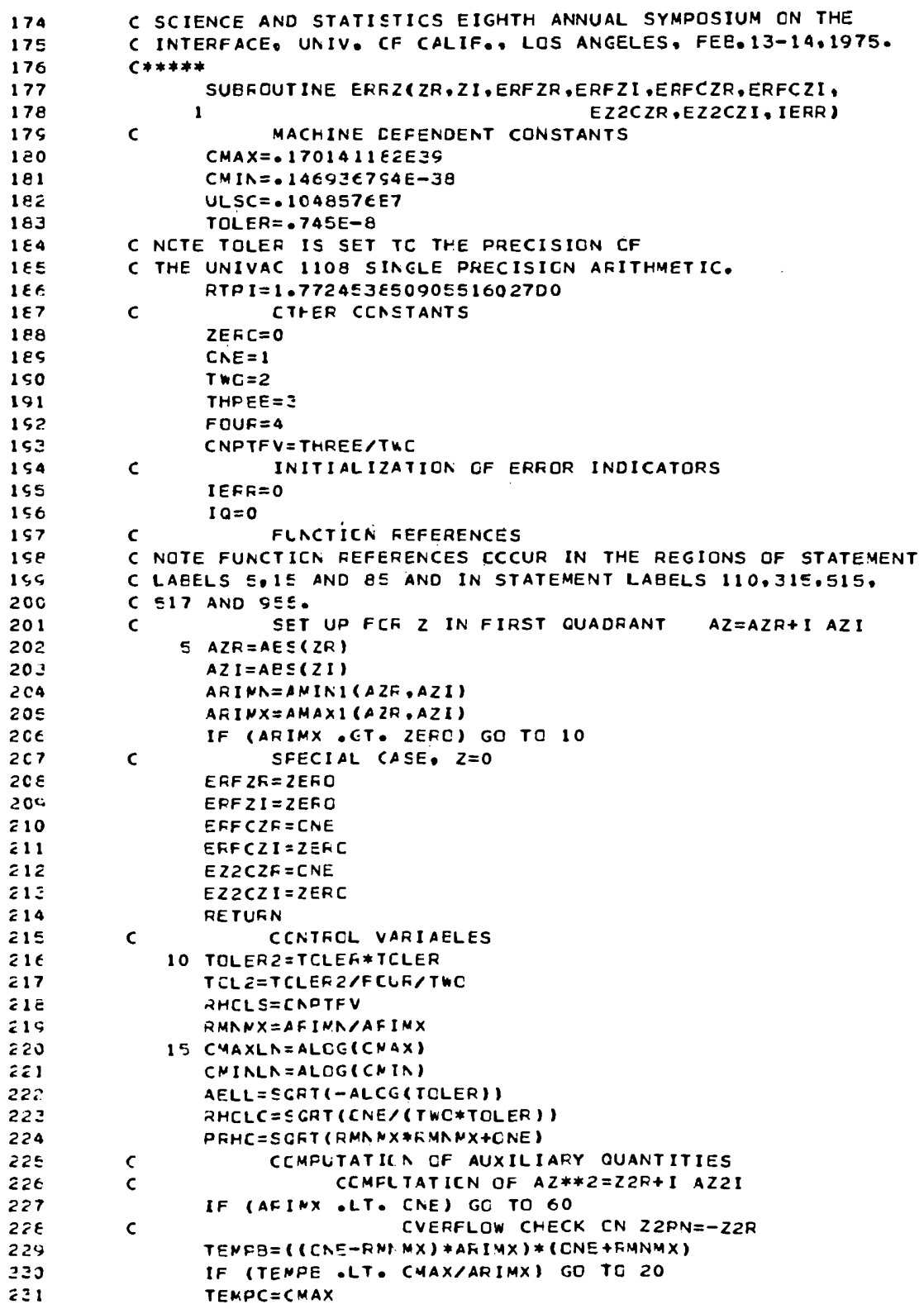


GO TO 30

20 TEMFC $=$ TEMPE*AFI $* X$

30 IF $(A Z F-A R I M X) \leq 0.40 .50$

40 Z2RA $=-T E N P C$ GC TC 70

SO $Z 2 F A=T E M F C$ GO TO 70

$60 Z 2 R A=(A Z I+A Z R) *(A Z I-A Z R)$ GC TC 90

C

70 IF (ARIMN ILT (CMAX/TWO)/ARIMX) GC TO BO $A Z 2 I=C N A X$ GO TC $\subseteq 0$

80 AZ2I=(TWO*ARINN)*ARIMX

C

c.

90 TENF $=22 F \Lambda / T H R E E$

$Z 2 R=-Z 2 F N$

IF TTENP OL $E M Z 2 D 3=C M A X$

$E Z 2 R=Z E F C$ GC TO 190

C

100 IF (TENP .CT. CMINLN) GO TO 110 EMZ2C $3=2 E F C$

c

I $a=1$ EXP(AZ**2) OVERFLOWS $(10=1)$

$110 E M Z 2 D E=E X P$ (TENF/TWO)

ENZ2D $=E M Z 2 D E * E N Z 2 D 6$

EZ2CE $=C N E / E M Z Z C E$

if (EZ2DE LE. CNE) GO TO 180

$J=1$

$P E X P=Z E F G$

$T E M F=E 2206$

120 IF (TEMP . EE. CMAX/EZ2D6) GO TO 150 TEMF $=$ TEMF $* E Z 2 C 6$

$J=J+1$

IF $(J-5) 1<0.130 .140$

130 PEXP $=$ TEMP GC 70120

140 EZ2F $=$ TEMF GO TO 190

150 IF (PEXP) $170,160.170$

160 PEXF $=C N A X$

$170 \quad E Z 2 R=C M A X$ GO TO 190

180 PEXP=E 2206*\%5 $E Z 2 R=P E X P * E Z 2 C E$ $T E Z 2=(P E X P * T W C) * E Z 2 D E$ 
190 IF (ARINX ILT CMAX/PRHO) GO TO 200 RHC $=$ C $M A X$

GC TC 210

c.

200 RHC $=A F I N X * P F H C$

c

210 IF (RHE LT. FHCLC) GO TO 220

$F A=A Z F / A F I M X$ IMFFCVE ACCURACY FOR LARGE RHD

$F B=A Z I / A R I M X$

$F C=R T F I *(R M N N X * R M N N X+O N E)$

$E Z 2 C Z F=(F A / A R I M X) / F C$

$E Z 2 C Z I=-(F E / A F I M X) / F C$ GO TO EOO

220 IF (RHC ILT FHCLS) GO TO 300

IF (AZF . GT. CNE) GC TO 500

IF (R+C-AELL) $300,700,700$

$c$

300 SUNF $=Z E F C$ PCWEF SERIES FOR ERF (AZ)

SUMI $=Z E F C$

$S E N=C N E$

$R N=Z E F C$

$D N=C N E$

PTMF $=A Z R$

C PTMI $=A Z I$

310 TNR=FTNR/CN

$T N I=F T I / C N$

$S U M F=S U M R+T N R * S G N$

SUM $1=S U M I+T N I * S G N$

C

$c$

SCALING TO AVOIC OVERFLOW OR UNDER-

I15 TMAX =AMAXI (AES (TMR), AES (TMI), ABS(SUMR), AES(SUMI))

IF (TMAX) $\equiv 20,360,320$

$320 T N N 2=(T M F / T N A X) * * 2+(T N I / T M A X) * * 2$

SUMNE $=($ SUMR $/ T M A X) * * 2+($ SUMI $/$ TMAX) $* * 2$

IF (TNM2) $\Xi \Xi 0, \Xi 60.330$

330 IF (SUNM2) $\$ 40.350 .340$

$340 \quad$ RENZ $=$ TMME $/ S L N N Z$

c TOLERANCE CHECK

C

IF (REMZ LLT. TCLER2) GC TO 360

$350 \quad D A=D A+T W C$

ACDITIONAL TERMS

$F N=F N+C N E$

$S G N=-S G N$

TEMF $=(F T M R * 22 F-P T M I * A Z 2 I) / R N$

PTNI $=$ (FTMF*AZZI +PTMI*Z2A) /RN

PTMR $=$ TEMP

GC TC $\$ 10$

c

3EO ERFZR=SUMF*TWC/FTPI

ERFZI=SUMI*TWC/FTPI

EFFCZF $=$ CNE-EFF $Z$ F

ERFCZI $=-E R F Z I$

$E Z 2 C Z F=(C C A Z 21 * E R F C Z R-S I A Z 21 * E R F C Z I) * E Z 2 R$

EZ2CZI=(SIAZZI*ERFCZR+CUAZ2I*EFFCZI) EZ 2 P

GC TD $\$ 40$

C

CCNTINUED FPACTION FOR EXP(AZ*¥Z)*EFFC(AZ) 


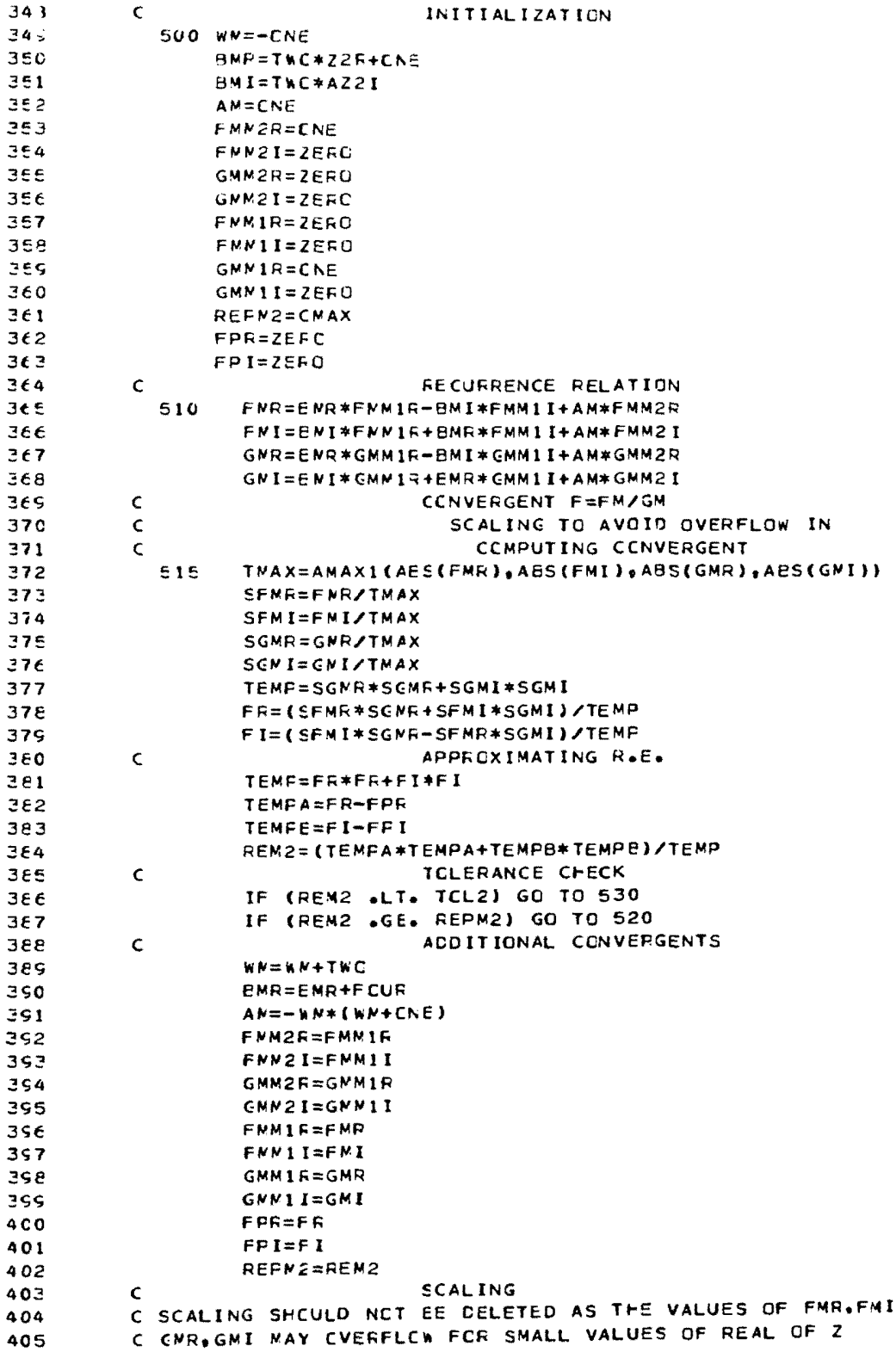




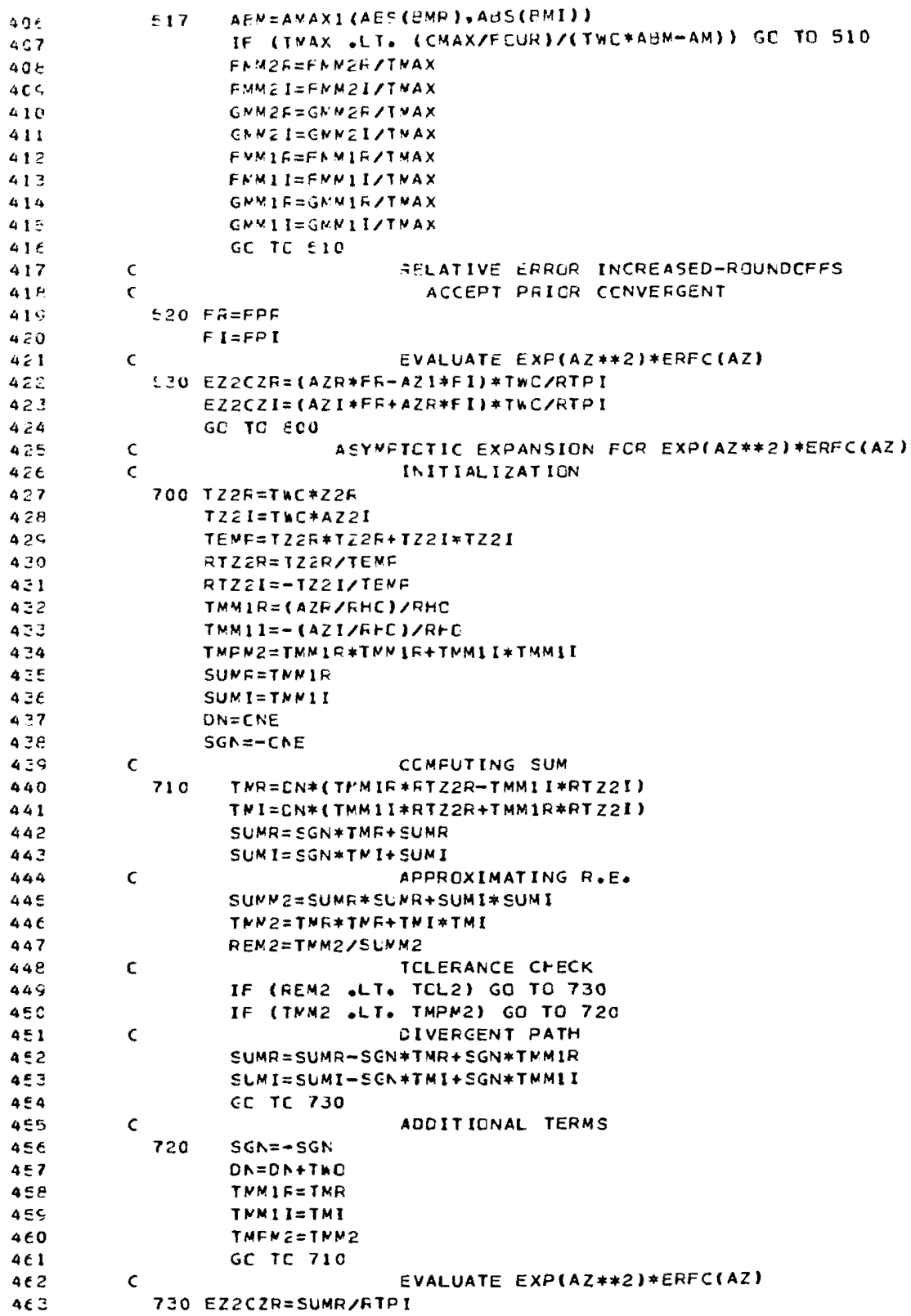




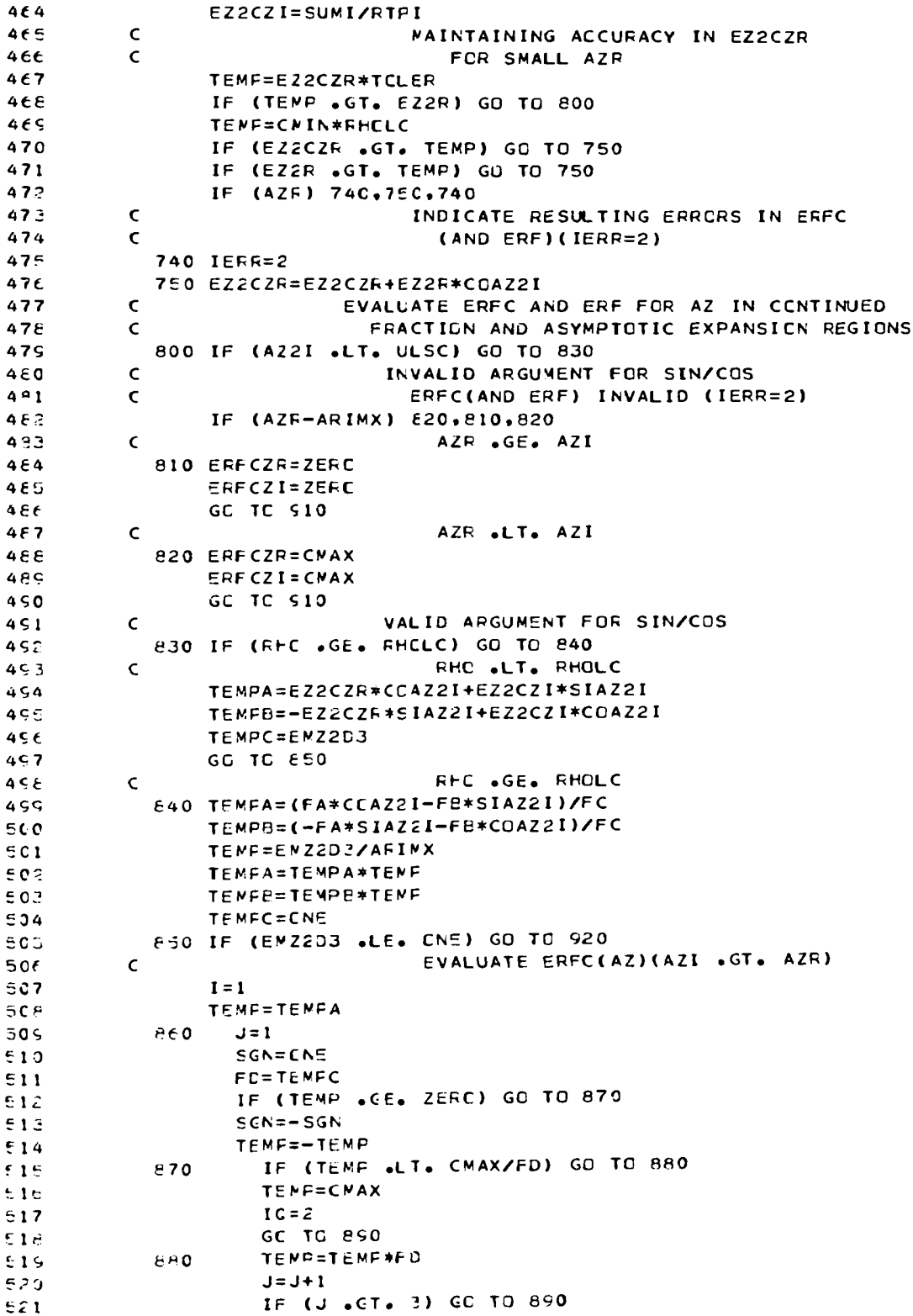




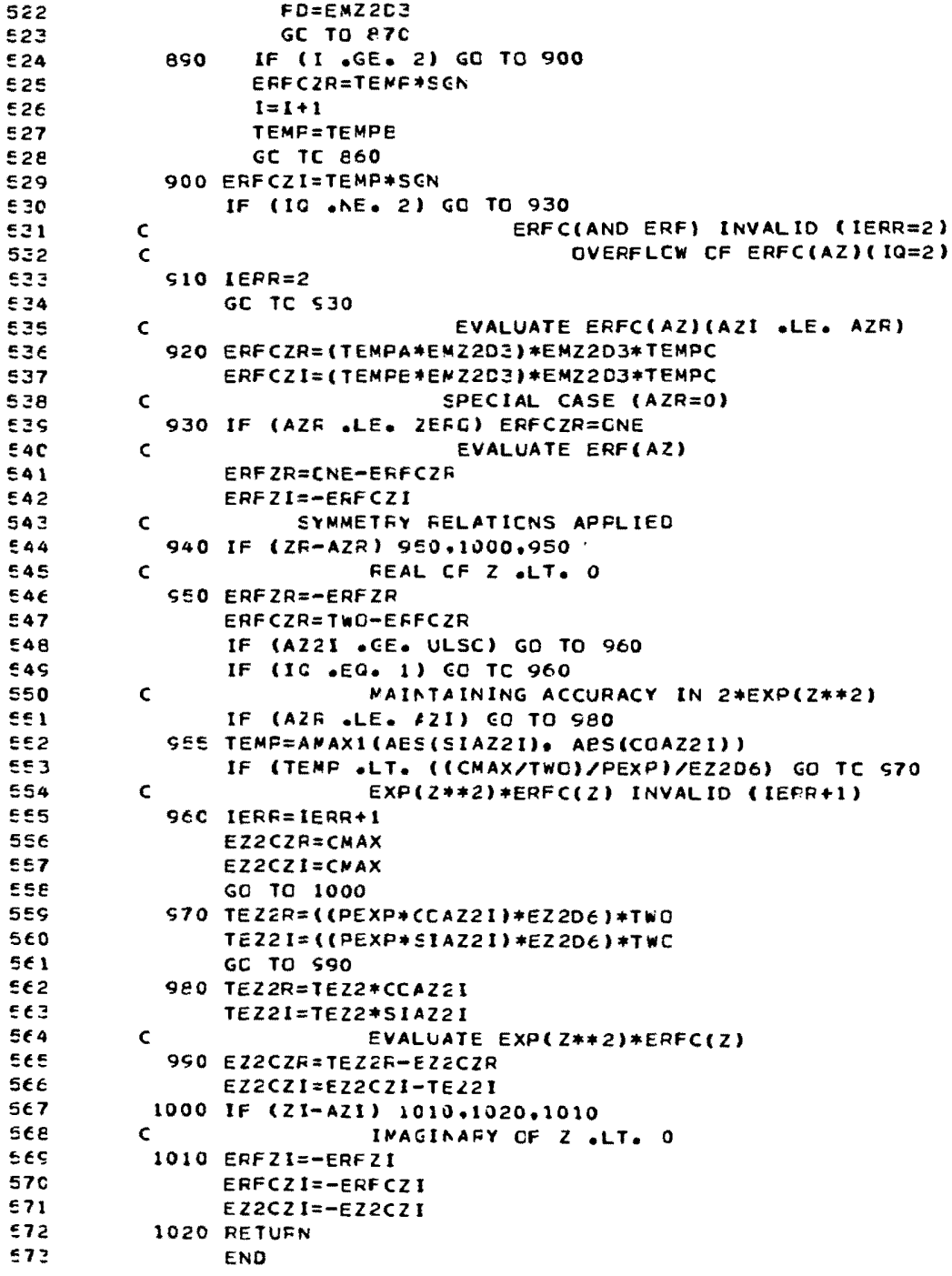


$15^{\circ}$

$.100000+01.000000$ $.978204+00-.583879-02$ $.956420+00-.116648-01$ $.934662+00-.174654-01$ $.912942+00-.232279-01$ $.891273+00-.289397-01$ $.869667+00-.345886-01$ $.848138+00-.401625-01$ $.826697+00-.456493-0$ $.805358+00-.510376-01$

$.784132+00-.563161-01$ $.680129+00-.806921-01$ $.580740+00-.100871+00$ $.487320+00-.115988+00$ $.487320+00-.115988+00$ $.401058+00-.125565+00$
$.322926+00-.129533+00$ $.322926+00-.129533+00$
$.253631+00-.128213+00$ $.193577+00-.122258+00$ $.142847+00-.112577+00$ $.101201+00-.100228+00$ $.680972-01-0863111-01$ $.680972-01-.063111-01$ $.427427-01-.718743-01$ .241557-01 -.578252-01 - $112438-01$-.448760-01 .288326-02 -.335145-01 $-.200643-02-.240031-01$ $-.440212-02-.164025-01$ $-.513475-02-.106123-01$ -.486777-02 -.641967-02 -.409504-02 -.354079-02 -.409504-02 -.0354979-02 $-.315468-02$
$-.0225377-02$
$-.0621965-03$ $-.225377-02$
$-.149843-02-021965-03$
$-.0981389-04$

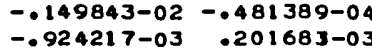
$-.523179-03.266848-03$ $-.265334-03.241454-03$ -.113783-03 .183127-03 $-.339435-04 \cdot 122861-03$

$.198248-05 \quad .741051-04$ $.383634-05-.331302-06$ $-.506480-07-.121829-06$ $-.172664-08 \quad .241877-08$ .431344-10 -.812606-11 .222076-14.150084-14 $-0005982-17-0633184-17$ . $.276523-19 \quad 109112-19$ $-.517996-22 \quad .562585-23$ .396093-25 -.443759-25 $.111560-28 \quad .427748-2$ $-.213063-31-.174915-32$ $30^{\circ}$

$.100000+01.000000$ $.980456+00-.112808-01$ $.960912+00-.225435-0$ $.941368+00-.337702-01$ $.921824+00-.449428-01$ $.902280+00-.560434-01$ $.882738+00-.670542-01$ $.863197+00-.779575-01$ $.843657+00-.887357-0$ $.824121+00-.993713-01$

$.804590+00-.109847+00$ $.707071+00-.159241+00$ $.610081+00-.202202+00$ $.514270+00-.236937+00$ $.420627+00-.261983+00$ $.330478+00-.276308+00$ $.245436+00-.279418+00$ $.167296+00-.271437+00$ $.978859-01-.253165+00$ $.388720-01-.226077+00$ $-.844458-02-.192259+00$ $-.433244-01-.154271+00$ $-.657413-01-.114934+00$ -.764609-01 -. $770708-01$ $-.770202-01-.432136-01$ $-.696000-01-.153368-01$ -.567576-01 .535338-02 $-.413301-01 \quad .185261-01$ -.257133-01 047077-01 - 119733-01 01 -.144281-02 .215037-01 $.531949-02.156622-01$ $.847425-02 \quad .930477-02$ $.872881-02.372996-02$ $.709056-02-.293708-03$ $.460569-02-.253794-02$ $.214179-02-.321372-02$ $.256793-03-.279917-02$

$-.830403-03-.184961-02$ $.361438-04.343118-03$ $-.100583-04-.454411-04$ $.342245-05.358814-05$ $. .410390-06.690194-07$ -.988939-10 - .274562-07 $.136924-08-.383336-09$ .477805-10 -324007-10 $.941894-12$.157585-11 $.233092-13.393021-13$ $.731135-15$.506747-15 $.130972-16-.343079-17$ $.313042-20-.160996-18$ $37.5^{\circ}$

$100000+01.000000$ $.982095+00-.137355-01$ $.964183+00-.274543-01$ $.946257+00-.411398-01$ $.928310+00-.547751-01$ $.910337+00-.683437-01$ $892330+00-818288-01$ $874283+00-.952136-01$ $.856190+00-.108481+00$ $.838045+00-.121615+00$

$.819844+00-.134598+00$ $.727824+00-.196662+00$ $.633845+00-.252418+00$ $.537866+00-.299764+00$ $.440342+00-.336667+00$ $.342317+00-.361274+00$ $.245465+00-.372052+00$ $.152078+00-.367979+00$ $.649787-01-.348749+00$ $-.126659-01-.314972+00$ - $776217-01-0260341+00$ $-.126958+00-.211694+00$ $-.158478+00-.148963+00$ $-.171150+00-.849352-01$ $-.165475+00-.248347-01$ -.143694+00 .262601-01 -.109748+00 .641075-01 -.689521-01 .859866-01 -.273484-01 .912368-01 $.916428-02.815039-01$ $.358034-01 \quad .605788-01$ $.497620-01.337876-01$ $.507367-01.700451-02$ $.409438-01$-.145264-01 .245557-01 -.272819-01 $.664754-02-.301636-01$ $-.809354-02-.245881-01$ -.165397-01 -.138997-01

-.178141-01-.225516-02 $.663124-02.774278-03$ $-.206887-02.808579-03$ $.149447-03-.641621-03$ $.163226-03.593711-04$ $.529439-06.406179-04$ -.550781-05 .636094-05 - $126107-050686874-06$ - 164519-06 $187753-06$ $.525818-10.356932-07$ $.420606-08 \quad .160997-08$ $.123912-09-.485650-09$ $-.462157-10 \quad .168052-10$ $45^{\circ}$ $.984040+00-.159556-01$ $.968068+00-.318984-01$ $.952070+00-.478156-01$ $.936033+00-.636943-01$ $.91954 C+00-.795217-01$ $903796+00-.952846-01$ $887571+00-.110970+00$ $.871258+00-.126564+00$ $.854845+00-.142053+00$

$.838321+00-.157424+00$ $.753652+00-031995+00$ 0
0 $470518+00=.415645+00$ $.365070+00-.455585+00$ $.255106+00-.480414+00$ $.142621+00-.487404+00$ $.307358-01-.474148+00$ $-.762502-01-.438939+00$ $-.172938+00-.381252+00$ $-.253111+00-.302268+00$ $-.310250+00-.205369+00$ $-.338390+00-.965018-01$ $-.333094+00$. 157600-01 $-.292775+00 \cdot 120601+00$ $-.219843+00 \quad .205946+00$ $-.121580+00.260096+00$ $-.103117-010273926+00$ 974065-01 $0143355+00$ $183390+00 \quad .171536+00$ $230486+00 \div 700421-01$ $.227631+00-.417236-01$ $.173531+00-.139462+00$ $.792740-01$-. $199249+00$ $-.321336-01-.204295+00$ $-.130194+00-.151392+00$ $-.185124+00-.545715-01$

$-.178018+00 \quad .564096-01$ $.146013+00-.667835-01$ $-.704373-01 \quad 0121816+00$ $-.697054-01-.103987+00$ $.909031-01-.666628-01$ .945377-01.396363-01 $.563713-01.752047-01$ $.498547-01$ 0710156-01 $0710858-010379415-01$ $-.258232-01-.056143-01$ $-.475602-01.462887-01$ $.623821-01-.612171-02$ 


\section{$\operatorname{ERFC(Z)}$}

$60^{\circ}$

RHOITHETA $50^{\circ}$

$.00 \quad 100000+01 \quad .000000$

$.02 \quad .985491+00-.172863-01$

$.04 .070967+00-.345635-01$

$.06 \quad .956411+00-.518226-01$

. $08 \quad .941809+00-.690545-01$

$.10 \quad .927144+00-.862497-01$

$.12 \quad .912401+00-.103399+00$

$.14 \quad .897565+00-.120493+00$

$.18 \quad .867552+00-.154473+00$

$.20 \quad .852345+00-.171339+00$

$.30 \quad .773712+00-.253982+00$

$.40 \quad .689468+00-.332643+00$

$.50 \quad .558041+00-.405420+00$

$.60 \quad .498195+00-.469950+00$

$.70 \quad .389205+00-.523342+00$

$.80 \quad .271098+00-.562163+00$

$.90 \quad .144933+00-.582512+00$

$1.00 \quad .131385-01-.520211+00$

(1)

$1.20-.248882+00-.491928+00$

$1.30-.364829400-.400481+0$

$1.40-.457890+00-.277246+00$

$1.50-.516623+00-.126215+00$

$1.60-.529455+00 \quad .439353-01$

$1.80-.382619+00 \quad .379468+00$

$1.90-.219614+00-37968+00$

$1.90-.519614+00 \quad .501 \mathrm{c81}+00$

$2.00 \quad-.101646-01 \quad .558267+00$

$2.10 \quad .220530+00 \quad .528666+00$

$2.20 \quad .434783+00 \quad .399806+00$

$2.30 \quad .586112+00 \quad .176582+00$

$2.40 \quad .627560+00-.112358+00$

$2.50 \quad .524677+00-.412231+00$

$2.60 \quad .271303+00-.647141+00$

$2.70-.961516-01-.735157+00$

$2.80-.492661+00-.613886+00$

$2.90-.794447+00-.271241+00$

$3.00-.870118+00 \quad .227570+00$

$3.50 \quad .128375+01-.438817+00$

$4.00 \quad-.143233+01 \quad .176970+01$

$4.50-.153046+01-.394580+01$

$5.00 \quad .818979+01-.289861+01$

$\begin{array}{lll}5.00 & .818979+01 & -.289861+01 \\ 5.50 & .141149+02 & .136673+02\end{array}$

$6.00 \quad .892289+01 \quad .480481+02$

$6.50 \quad .767743+01 \quad .133290+03$

$7.00 \quad .164446+03 \quad .364872+03$

$7.50 \quad .126058+04 \quad .375323+03$

$8.00 \quad .231199+04-.413300+04$

$8.50-.181429+05-.441024+04$

9.00
$.100000+01.000000$

$.988713+00-.195441-01$

$977408+00-.390882-0$

$.966067+00-.586322-0$

$.954672+00-.781761-0$

$.943204+00-.977195-01$

$.931646+00-.117262+00$

$.919978+00-.136803+00$

$.908183+00-.156342+00$

$.896242+00-.175878+00$

$884135+00-.155409+00$ $820454+00-.292919+00$ $.749698+00-.389843+00$ $.669242+00-.485367+00$ $.576286+00-.578076+00$ $.467898+00-.665719+00$

$.341135+00-.744933+00$

$.193283+00-.810912+00$

$222736-01-057061+00$

. $172655+00-0874670+00$

$.389989+00-.852699+00$

$-.624572+00-.777828+00$

$-.865837+00-.635009+00$

$-.109565+01-.408856+00$

$-.128600+01-.863300-01$

$-.139708+01 \quad .338770+00$

$-.137659+01 \quad .859011+00$

- $116196+01 \quad 144402+01$

$-.687539+00 \quad .202914+01$

$.100155+00 \quad .250402+01$

$.121649+01.270506+C 1$

$.260024+01 \quad .241951+01$

$.406480+01 \quad .141207+01$

$.524907+01-.511462+00$

$.559076+01-.339050+01$

$.436233+01-0.694257+01$

$07373+01-0.103663+02$

$-.542122+01-121876+02$

$-.139065+02-.102944+02$ $.436770+02.609546+C 2$ $-.286672+03-.315634+03$

. $303475+04.899044+03$ $-.236016+05 \quad .194203+05$ $-.192298+06-.331209+06$ $.434927+07-.444020+07$ $.130111+09.950077+07$ - $3 \operatorname{cog} 19+10 \quad .172358+10$ $.107926+12.606848+11$ $.557097+13.462008+12$ $.231401+15-.228725+15$ $-.117844+17-.213680+17$ $70^{\circ}$

$.100000+01.000000$

$.952279+00-.212081-01$

$.984542+00-.424252-0$

$.976774+00-.036604-01$

. $968559+00-.849226-01$

$.961080+00-.106221+00$

$.953123+00-.127564+00$

$.945070+00-.148961+00$

$.936506+00-.170421+00$

$.928612+00-.191952+00$

$.920173+00-.213564+00$

$.875153+00-.323124+00$

$.823615+00=.435932+00$

$.762712+00-.552889+00$

$.688562+00-.674665+00$

$.598036+00-.801524+00$

$.484501+00-.933057+00$

$.341532+00-.106775+01$

$.160625+00-0120233+01$

$-.686592-01-.133079+01$

$-.358812+00-.144298+01$

$-.724161+00-.152248+01$

$-.118000+01-.154380+01$

$-.174051+01-.146840+01$

$-.241452+01-.123975+01$

$-.319777+01-.777391+00$

$-.405945+01 \quad .291660-01$

$-.452009+01 \quad .132454+01$

$-0561710+01.320529+01$

$-.585370+01 \quad .610278+01$

$-.512891+01 \quad .993582+01$

$-.265168+01 \cdot 148074+02$ $.274334+01.204062+02$ . $126293+02 \quad .257378+02$ $.288616+02.285642+02$ . $530807+02.245883+02$ . $554677+020.044245+01$ $.122167+03-.371981+02$

$.150602+03-.121463+03$ $-.185185+04-.696951+03$ -142504+05.268505+05 $-.581405+05-.694896+06$ $-.301677+05 \quad .237891+08$ $-.279467+09-.117057+10$ $.640472+11.633707+11$ $-.9 \$ 2019+13 \quad .967322+12$ $.442269+15-.156615+16$ $.371118+18 \cdot 125665+18$ $-.785527+19 \cdot 138783+21$ $-.624892+23.370071+23$ $-.554262+26-.688648+25$ $80^{\circ}$

$.100000+01 \quad .000000$ $.996080+00-.222273-01$ $092150+00-.444703-01$ $.988203+00-.667446-01$ $.984228+00-.890659-01$ $.980217+00-.111450+00$ $.976160+00-.133913+00$ $.972048+00-.156471+00$ $.967870+00-.179140+00$ $.963617+00-.201936+00$

$.959279+00-.224877+00$ $.935924+00-.342344+00$ $.908660+00=.466059+00$ $.875613+00-.598673+00$ $.834330+00=.742997+00$ $.781501+00-.742997+00$ $.712565+00=.108143+01$ $.621138+00-.128468+01$ $.498184+00-0151831+01$ . $330784+00-0178947+01$ $.100318+00-.210652+01$ $-.220245+00-.247879+01$ $-.670390+00-.291589+01$ $-.130809+01-.342593+01$ $-.221880+01-.401186+01$ $-.352885+01-.466405+01$ $-.542509+01-.534607+01$ $-.818351+01-0.596797+01$ - $122098+02-0633691+01$ $0.006086+01$ $-266833+02-.441546+01$ -.391538+02 $.862691-02$ $-.570679+02 \quad .976572+01$ $-.823162+02 \quad .294995+02$ $-.116747+03 \quad .675244+02$ $-161012+03.138532+03$ $011587+03$ $-.217771+03040985+03$

$-.246068+03 \quad .906353+03$ $.126726+05 \quad .110011+05$ $.414164+06-.267047+06$ $-.104472+08-.211627+08$ $-.159723+10 \quad .902680+09$ -155714t12 $1170313+1$ $-196750+14-.42 .5327+14$ $-.152018+17 \quad .205488+16$ $.619376+19 \quad .519553+19$ $-.143772+22-.670033+22$ $-.257275+25 \quad .897512+25$ $.115166+29-.168785+29$ $-.488030+32 \quad .526592+32$
$1+01$ $.1+01$ $-1+01$ $1+0$ $-1+01$ $-1+01$ $-1+01$ $1+0$ $-1+01$ $-1+01$

$-1+01$ $-1+01$ $-1+01$ $-1+01$ $-1+0$ $-1+0$ $1+0$ $\because 1+01$ $1+0$ $1+0$ $01+01$ $-1+01$ $1+0$ $-1+0$ $-1+0$ $-1+01$ $-1+0$ $.1+01$ $.1+0$ $-1+0$ $-1+0$ $-1+0$ $-1+0$ $-1+0$ $-1+0$ $-1+0$ $-1+01$ $-1+0$ $1+0$ $-1+0$

$.1+01$ $-1+0$ $-1+0$ $-1+01$ $1+01$
$.1+01$ $-1+01$ $-1+01$ $-1+0$ $-1+01$ $-1+0$ $-1+0$ $-1+01$

$.1+01$

$90^{\circ}$

.000000

-.225706-01 -.451593-01 -.677841-01 -.904633-01 - $113215+00$ $-.136058+00$ - $159011+00$ -.182093+00

$-.205323+00$

$-.228721+00$ $-.348949+00$ $-.476625+00$ $-.614952+00$ $-.767853+00$ $-.940283+00$ -.137154+01 - $165043+01$ -.241591+01 $-.295609+01$ -.365696+01 


\begin{tabular}{|c|c|c|}
\hline \multicolumn{2}{|c|}{ RHOITHETA } & \multirow{2}{*}{$0^{\circ}$} \\
\hline & $.100000+01$ & \\
\hline .02 & $.977826+00$ & .0 \\
\hline .04 & $.956418+00$ & .0 \\
\hline .06 & $.935741+00$ & .0 \\
\hline .08 & $.915764+00$ & .0 \\
\hline -10 & $.896457+00$ & .0 \\
\hline .12 & $.877791+00$ & .0 \\
\hline .14 & $.859740+00$ & .0 \\
\hline .16 & $.842277+00$ & .0 \\
\hline .18 & $.825378+00$ & .0 \\
\hline .20 & $.809020+00$ & .0 \\
\hline • 30 & $.734559+00$ & .0 \\
\hline .40 & $.670788+00$ & .0 \\
\hline .50 & $.615690+00$ & .0 \\
\hline .60 & $.567805+00$ & .0 \\
\hline .70 & $.525930+00$ & .0 \\
\hline $\begin{array}{l}.80 \\
.90\end{array}$ & $.489101+00$ & .0 \\
\hline $\begin{array}{r}.90 \\
1.00\end{array}$ & $.456532+00$ & .0 \\
\hline $\begin{array}{l}1.00 \\
1.10\end{array}$ & .42758 & .0 \\
\hline $\begin{array}{l}1.10 \\
1.20\end{array}$ & $.401730+00$ & $\cdot 0$ \\
\hline $\begin{array}{l}1.20 \\
1.30\end{array}$ & $\begin{array}{r}378537+00 \\
357643+00\end{array}$ & $\cdot 0$ \\
\hline 1.40 & $338744+00$ & .0 \\
\hline 1.50 & $.321585+00$ & .0 \\
\hline 1.60 & $.305953+00$ & .0 \\
\hline 1.70 & $.291663+00$ & .0 \\
\hline 1.80 & $.278560+00$ & .0 \\
\hline 1.90 & $.266509+00$ & .0 \\
\hline 2.00 & $.255396+00$ & $\cdot 0$ \\
\hline 2.10 & $.245119+00$ & .0 \\
\hline 2.20 & . $235593+00$ & .0 \\
\hline 2.30 & $.226742+00$ & .0 \\
\hline 2.40 & $.218499+00$ & .0 \\
\hline $2 \cdot 50$ & $.210806+00$ & .0 \\
\hline 2.60 & $.203613+00$ & .0 \\
\hline $2 \cdot 7$ & $-196874+00$ & $\cdot 0$ \\
\hline $2 \cdot 80$ & $190549+00$ & .0 \\
\hline 2.90 & $.184602+00$ & .0 \\
\hline 3.00 & $.179001+00$ & .0 \\
\hline 3.50 & $.155294+00$ & .0 \\
\hline 4.00 & $136999+00$ & .0 \\
\hline 4. & - $122485+00$ & $\cdot 0$ \\
\hline 5.00 & $.110705+00$ & .0 \\
\hline $5 \cdot 50$ & $-100962+00$ & $\bullet 0$ \\
\hline 6.00 & -927766-01 & 0 \\
\hline 6.50 & $.858057-01$ & $\bullet 0$ \\
\hline .00 & -798001-01 & - 0 \\
\hline & $\begin{array}{r}745737-01 \\
045852-01\end{array}$ & 0 \\
\hline & $.659251-01$ & .0 \\
\hline .00 & $.623077-01$ & $\bullet 0$ \\
\hline
\end{tabular}

$15^{\circ}$

$.100000+01 \quad .000000$

$.978544+00-.564511-02$ $.957755+00-.109148-01$ $.937610+00-.158323-01$ $.918086+00-.204192-01$ $859159+00-.246960-01$ $.880810+00-.286817-01$ $.863016+00-.323942-01$ $.845758+00-.358502-01$ $.829017+00-.390656-01$

$.812775+00-.420551-01$ $.738441+00-.540691-01$ $.674177+00-.621855-01$ $.618355+00-.674592-01$ $.569635+00-.706620-01$ $.526911+00-.723596-01$ $.489272+00-.729681-01$ $.455962+00-.727939-0$ $.426354+00-.720633-01$ $.399923+00-.709434-01$ $.376233+00-.695576-01$ $.354916+00-.679971-01$ $.335662+00-.663291-0$ $.318209+00-.646029-0$ $.302334+00-.628545-01$ $.287848+00-.611100-0$ $.274589+00-.593879-0$ $.262415+00-.577015-0$ $.251208+00-.560596-01$ $.240863+00-.544681-0$ $.231289+00-.529306-01$ $.222408+00-.514490-0$ $.214150+00-.500239-01$ $.206456+00-.486549-0$ $.199271+00-.473413-01$ $.152549+00-.460815-01$ $.186248+00-.448740-01$ $.180331+00-.437168-01$

$174766+00-.426079-0$ $.151281+00-.377161-0$ $.133241+00-.337346-0$ $.118978+00-.304579-01$ $.107435+00-.277278-0$ $.979088-01-.254257-01$ $831244-01-.217720-01$ $.772774-01-.203020-01$ $.721940-01-.190134-01$ $.677345-01-.178753-0$ $.637913-01$-.168633-01 $.602800-01-.159579-01$ $30^{\circ}$

$.100000+01 \quad .000000$

$.980656+00-.109433-01$ $.961711+00-.212290-01$ $.943165+00-.308907-01$ $.925014+00-.399605-01$ $.907257+00-.484692-01$ $.889890+00-.564458-01$ $.872908+00-.639183-01$ $.856308+00-.709132-01$ $.840085+00-.774557-01$

$.824233+00-.835698-01$ $.750340+00-.108476+00$ $.684212+00-.125660+00$ $.626815+00-.137032+00$ $.575515+00=.144060+00$ $.530125+00-.147863+00$ $.489917+00-.14929 E+00$ $.454239+00-.149005+00$ $.422513+00-.147478+00$ $.394231+00-.145081+00$ . $368 \$ 49+00-.142050+00$ $.346284+00-.138710+00$ $.325505+00-.135093+00$ $.307525+00-.131350+00$ $.290896+00-.127564+00$ $.275808+00-.123794+00$ $.262075+00-.120081+00$ $.249540+00-.116455+00$ $.238065+00-.112935+00$ $.227532+00-.109534+00$ $.217837+00-.106258+00$ $.208891+00-.103112+00$ $.200615+00-.100095+00$ $.192541+00-.972068-01$ $.185809+00-.944437-0$ $.179166+00-.918020-01$ $.172967+00-.892774-01$ $.167168+00-.868650-01$

$161735+00-.845599-0$ $139036+00-.744669-0$ $.121846+00-.663430-0$ $.108404+00-.597166-0$ $.976147-01-.542350-01$ $.887699-01-.496392-01$ $813899-01-.457386-01$ $.751399-01-.423911-01$ 697797-01 -.394900-0 $.651322-01$-. 369532-0 $.610646-01-.347176-01$ $.574747-01-.327332-01$ $.542832-01$-.309606-01 $37.5^{\circ}$

$.100000+01 \quad .000000$ $.982202+00-.133574-0$ $.964623+00-.259749-01$ $.947276+00-.378843-01$ $.930171+00-.491167-01$ $.913315+00-.597018-01$ $.896717+00-.696688-01$ $.880383+00-.790455-0$ $.864318+00-.878590-0$ $.848526+00-.961353-01$

$.833010+00-.103900+00$ $.759640+00-.135855+00$ $.693269+00-.158259+00$ $.633647+00-.173298+00$ $.580342+00-0182718+00$ $.532829+00-.187900+00$ $.490547+00-.189926+00$ $.452940+00=1189631+00$ $.419480+00-.187662+00$ $.389679+00-.184510+00$ $.363094+00-.180548+00$ $.339332+00-.176056+00$ $.318044+00-.171244+00$ $.298923+00-.166267+00$ $.281701+00-.161236+00$ $.266147+00-.156235+00$ $.252058+00-.151319+00$ $.239260+00-.146528+00$ $.227600+00-.141889+00$ $.216948+00-.137417+00$ $.207188+00-.133122+00$ $.198222+00-.129008+00$ $.189963+00-.125073+00$ $.182336+00-.121315+00$ $.175275+00-.117730+00$ $.168723+00-.114311+00$ $.162630+00-.111051+00$ $.156950+00-.107943+00$

$.151645+00-.104980+00$ $.129658+00-.920841-01$ $.113196+00-.817946-01$ $.100432+00-.734593-01$ $.902530-01-.666012-01$ $.819492-01-.608758-01$ 750469-01-.560327-01 $.692192-01-.518878-01$ $642331-01-.483034-01$ $.599186-01-.451749-01$ $.561483-01-.424219-01$ $.528254-01-.399814-01$ $.498745-01-.378036-01$ $45^{\circ}$

$.100000+01.000000$ $.984046+00-.155619-01$ $.968117+00-.303494-01$ $.952236+00-.443878-01$ $.936422+00-.577025-01$ $.920696+00-.703184-01$ $.905075+00-.822605-01$ $.889575+00-.935532-01$ $.874212+00-.104221+00$ $.858998+00-.114286+00$

$.843946+00-.123774+00$ $.771453+00-.163319+00$ $.704192+00-.191604+00$ $.642609+00-.210932+00$ $.586778+00-.223250+00$ $.536524+00-.230166+00$ $.491521+00-.232989+00$ $.451358+00-.232766+00$ $.415588+00-.230320+00$ $.3 \varepsilon 3760+00-.226295+00$ $.355440+00-.221186+00$ $.3 \geq 0223+00-.215369+00$ $.307738+00-.209128+00$ $.287653+00-.202672+00$ $.269672+00-.196156+00$ $.253535+00-.189690+00$ $.239013+00-.183351+00$ $.225508+00-.177191+00$ $.214048+00-.171246+00$ $.203282+00-.165535+00$ $.193480+00=.160068+00$ $.184530+00-.154849+00$ $.176334+00-.149876+00$ $.168808+00-.145143+00$ $.161878+00-.140642+00$ $.155479+00-.136364+00$ $.149556+00-.132298+00$ $.144061+00-.128434+00$

$.138950+00-.124761+00$ $.117989+00-.108897+00$ $.102526+00-.063790-01$ $.906646-01-.863253-01$ $.812804-01-.781076-01$ $.736702-01$-.712820-01 $.673730-01-.655313-01$ 620749-01 -.606252-01 575547-01-.563934-01 $.536520-01-.527075-01$ $.502479-01$-.494694-01 $.472522-01-.466031-01$ $.445553-01-.440484-01$ 
RHONTHETA $50^{\circ}$

$.00 \quad .100000+01 \quad .000000$

$.02 .985430+00-.168969-0$

$.04 .970750+00-.330243-01$

$.06 \quad .955991+00-.484013-01$

$.08 \quad .941179+00-.630470-01$

$.10 \quad .926338+00-.769813-01$

$.12 .911494+00-.902242-01$

$.14 \quad .896667+00-.102796+00$

$.16 \quad .881878+00-.114717+00$

$.18 \quad .867146+00-.126007+00$

$.20 \quad .852490+00-.136687+00$

$.40 \quad 713036+00-.214204+00$

$.50 \quad .649979+00-.236926+00$

. 60 . $649979+00-.236926+00$

$.60 \quad .592156400-.251548+00$

$.70 \quad .539686+00-.259892+00$

$.80 \quad .492447+00-.263412+00$

$.90 \quad .450161+00-.263301+00$

$1.00 \quad .412460+00-.260519+00$

$1.10 .378934+00-.255830+00$

$1.20 .349161+00-.249826+00$

$1.30-322730+00-.242965+00$

$1.30 \quad .322730+00-.242965+00$

$1.40 \quad .299256+00-.235553+0$

$1.50 \quad .278384+00-.227969+00$

$1.60 \quad .259793+00-.220280+00$

$1.70 .243198+00-.212662+00$

$1.80 .228349+00-.205210+00$

$1.90 \quad .215025+00-.197986+00$

$2.00 \quad .203034+00-.191033+00$

$2.10 .192211+00-.184372+00$

$2.20 \quad .182411+00-.178015+00$

$2.30 \quad .173510+00-.171964+00$

$2.40 \quad .165400+00-.166214+00$

$2.50 \quad .157988+00-.160758+00$

$2.60 \quad .151195+00-.155584+00$

$2.70 \quad .144550+00-.150680+00$

$2.80 \quad .139193+00-.146031+00$

$2.90 \quad .133871+00-.141624+00$

$3.00 \quad .128940+00-.137445+00$

$3.50 \quad .108892+00-.119509+00$

$4.00 \quad .942797-01-.105481+00$

$4.50 \quad .831655-01=.942896-01$

$5.00 \quad .744259-01-.851875-01$

$5.50 \quad .673700-01-.776560-01$

$6.00 \quad .615511-01-.713293-01$

$6.50 \quad .566679-01-.659441-01$

$7.00 \quad .525101-01-.613075-01$

$7.50 \quad .489261-01-.572750-01$

$8.00 .458040-01-.537368-01$

$0.500 .430594-01-.506078-01$

8.50
$9.00 \quad .430594-01-.506078-01$
$.406273-01-.478214-01$ $60^{\circ}$

$.100000+01 \quad .000000$ $.988522+00-.191978-0$ $.976680+00=.377036-01$ $.964508+00-.555200-01$ $.952039+00-.726507-01$ $.939307+00-.891010-01$ $.926342+00-.104877+00$ $913175+00-.119987+00$ $899834+00-134439+00$ $.886348+00-.148243+00$

$.872742+00-.161410+00$ $.803774+00-.218107+00$ $.735131+00-.260834+00$ $.668830+00=.291462+00$ $606260+00-.311920+00$ $548281+00-.324060+00$ $.495329+00-.329575+00$ $.447520+00-.329960+00$ $.404741+00-.326488+00$ $.366725+00-.320215+00$ $.333111+00-.311994+00$ $.303494+00-.302503+00$ $.277452+00-.292262+00$ $.254575+00-.281666+00$ $.234476+00-.271004+00$ $.216798+00-.260483+00$ $.201224+00-.250247+00$ $.187470+00-.240389+00$ $.175289+00-.230965+00$ $.164467+00-.222006+00$ $.154819+00-.213521+00$ $.146185+00-.205507+00$ $.138430+00-.197951+00$ $.131439+00-.190836+00$ $.125111+00-.184138+00$ $.119364+00-.177833+00$ $.114124+00-.171899+00$ $.109330+00-.166309+00$

$.104930+00-.161041+00$ $.874505-01-.138780+00$ $.750909-01-.121741+00$ $.658787-01-.108356+00$ $.587346-01-.975919-01$ $.530232-01-.887581-01$ $.483467-01-.813834-0$ $.444434-01-.751362-01$ $.411335-01$-.697775-01 $.382898-01-.651309-01$ $.358190-01-.610637-01$ $.336516-01-.574741-01$ $.317343-01-.542828-01$ $70^{\circ}$

$.100000+01.000000$ $.991580+00-.209465-01$ $.983379+00-.413619-01$ $.974228+00-.612308-01$ $.964559+00-.805399-01$ $.954404+00-.992775-01$ $.943794+00-.117434+00$ $.932761+00-135001+00$ $921336+00-151972+00$ $.909551+00-168342+00$

$.897434+00-.184108+00$ $032921+00-.253864+00$ $.7 € 4351+00-.308806+00$ $.694712+00-.349876+00$ $.626410+00-.378484+00$ $.561255+00-.396308+00$ $.500496+00-.405125+00$ $.444890+00-.406682+00$ $.394786+00-.402602+00$ . $350217+00-.394324+00$ $.310985+00-.383080+00$ $.276740+00-.369883+00$ $.247038+00-.355543+00$ $.221396+00-.340682+00$ $.199324+00-325767+00$ $.180352+00-.311128+00$ $.164045+00=.296992+00$ $.150014+00-.283502+00$ $.137912+00-.270739+00$ $.127441+00-.258737+00$ $118345+00-.247499+00$ $.110409+00-.237007+00$ $.103450+00-.227227+00$ $.973162-01-.218118+00$ $.918212-01-.209636+00$ $.870395-01-.201733+00$ 827037-01 - .194364+00 $.788013-01-.187486+00$

$.752717-01-.181058+00$ $.617 \mathrm{CB8}-01-.154443+00$ $.525024-01-.134607+00$ $.458048-01-.119295+00$ $.406890-01$-.107125+00 $.366399-01-.972205-01$ $.333477-01-.890010-0$ $.306137-01$-.820692-01 $.283042-01-.761436-01$ $.263257-01-.710192-01$ $.246107-01-.665434-01$ .231091-01-.626001-01 $.217828-01-.590993-01$ $80^{\circ}$

$100000+01.000000$ $.995708+00-.220828-01$ $.990684+00-.438614-01$ $.984947+00-.653065-01$ $.978518+00-.863902-0$ $.971421+00-.107086+00$ $.963679+00-.127369+00$ $.955317+00=.147215+00$ $946360+00-166602+00$ $.936834+00-.185511+00$

$.926768+00-.203921+00$ $.869300+00-.287964+00$ $.802581+00-.357672+00$ $.730181+00-.412479+00$ $655426+00-.452678+00$ $581214+00-.479263+00$ $509895+00-.493727+00$ $.443222+00-.497872+00$ $.382368+00-.493623+00$ . $327976+00-.482883+00$ $.280243+00-.467415+00$ $.239021+00-.448772+00$ $203909+00-.428255+00$ $174350+00-.406909+00$ $149701+00-385528+00$ $.129300+00-.364688+00$ $.112501+00-.344773+00$ $.987098-01-.326022+00$ $.873971-01-.308553+00$ $.781048-01-.292400+00$ $.704459-01-.277541+00$ $.641001-01-.263913+00$ $.588065-01-.251431+00$ $.543547-01-.240002+00$ $.505775-01-.229528+00$ $.473424-01-.219915+00$ $.445450-01-.211075+00$ $.421033-01-.202926+00$

$.399530-01-.195396+00$ $.321214-01-.165001+00$ $.270928-01-.143006+00$ $.235275-01-.126308+00$ $.208412-01-.113170+00$ $.187325-01-.102546+00$ $.170271-01-.937703-01$ $.156161-01-.863935-01$ $.144275-01-.801029-01$ $.134115-01-.746733-01$ $.125322-01-.699379-01$ $.117632-01-.657709-01$ $.110848-01-.620752-01$ $90^{\circ}$

$100000+01 \quad .000000$ $.999600+00-.225616-01$ $.998401+00-.450871-01$ $.996406+00-.675405-01$ $.993620+00-.898862-01$ $.990050+00-.112089+00$ $.985703+00=.134113+00$ $.980591+00-.155925+00$ $.974725+00-.177491+00$ $.968119+00-.198777+00$

$.960789+00-.219753+00$ $.913931+00-.318916+00$ $.852144+00-.406153+00$ $.778801+00-0478925+00$ $.697676+00-.535713+00$ $.612626+00-.576042+00$ $.527292+00-.600412+00$ $.444858+00-.610142+00$ $.367879+00-.607158+00$ $.298197+00-.593761+00$ $.236928+00-.572397+00$ $.184520+00-.545456+00$ $.140858+00-.515113+00$ $.105399+00-.483227+00$ $.773047-01-.451284+00$ $.555762-01-.420388+00$ $.391639-01-.391291+00$ $.270518-01-.364437+00$ $.183156-01-340026+00$ $.121552-01-.318073+00$ $.790705-02-.298468+00$ $.504176-02-.281026+00$ $.315111-02=.265522+00$ $.193045-02-.251723+00$ $.115523-02-.239403+00$ $.682328-03-.228355+00$ .393669-03 - $218399+00$ $.222630-03-.209377+00$

$.123410-03-.201157+00$ $.478512-05-.168830+00$ $.112535-06-.145954+00$ $.160523-08-.128735+00$ $.138879-10-.115246+00$ $.728772-13-.104367+00$ $.231952-15-.953962-01$ $.447773-18-.878644-01$ $.524289-21-.814475-01$ $.372336-24-.759126-01$ $.160381-27-.710891-01$ $.419009-31-.668445-01$ $.663968-35-.630821-01$ 
$\operatorname{EXP}(Z * * 2) * \operatorname{ERFC}(-Z)$

RHOITHETA

-00 $\quad 100000+01.0$

$.02 .102257+01.0$

$.04 \quad .104678+01 \quad .0$

$.06 \quad .107147+01 \quad .0$

$.10 \quad .112364+01.0$

$.12 .115122+01.0$

$.14 \quad .117985+01 \quad .0$

$16 \quad 120958+01$

$.18 .124048+01$

$.20 \quad .127260+01$

$.30 .145375+01$

$.40 \quad .167623+01$

$-195236+01$

$.60 \quad .229885+01$

$.80 .0273870+01$

$.90 \quad .403928+01$

$.00 \quad .500898+01$

$1.10 \quad .630524+01$

$1.20 \quad .006285+01$

$1.30 \quad .104813+02$

$1.40 \quad .138599+02$

$1.50 \quad .186539+02$

$1.60 \quad .255657+02$

$1.70 .356950+02$

$1.80 \quad .507889+02$

$1.90 .736656+02$

$2.00 \quad .108941+03$

$2.10 \quad .164294+03$

$2.20 .252703+03$

$2.30 \quad .396460+03$

$2.40 \quad .634478+03$

$2.50 \quad .103581+04$

$2.60 \quad .172508+04$

$2.70 \quad .293094+04$

$.80 \quad .508022+04$

$2.90 \quad .898334+04$

$3.00 \quad .162060+05$

3.50

4.00

4.50

5.00

5.50

6.00

6.50

7.00

7.50

8.00

0.00 $15^{\circ}$ $.102215+010004525-02$ $104502+01.125170-01$ $.106863+01.194435-01$ - $109302+01.268548-01$ $.111821+01$.347829-01 $.114424+01.432622-01$ $.117112+01 \quad .523294-01$ $.119891+01.620234-01$ $.122763+0$

$.125731+01$ - $142149+0$ $.161572+01$ - $184573+01$ $.211791+01$ $.243899+01$ 241529+0 $.325121+01$ $374645+01$ $.429102+01$ $.485657+01$ - $538160+01$ $.574679+01$ $.573426+01$ $.496175+01$ $.077771+01$ - $277771+01$ $-108404+02$ -.268411 +02 $-.542404+02$ $-.995202+02$ $-.171913+03$ $-.283609+03$ $-.448599+03$ $3-.164667+03$ $-.967019+03-.532419+03$ $-126560+04-.124780+04$ $-.141486+04-.254465+04$

$-.102319+04-.474402+04$ $.759689+05-.127565+05$ $-.303148+06 \quad .206132+07$ $-.632047+08-.532606+08$ $.504457+10-.335304+09$ $-.398070+12 \quad .262492+12$ $.457872+14-.520744+14$ $-.100716+17 \quad .118452+17$ $.433489+19-0317887+19$ $-.283374+22 \quad .426347+2$ $.196497+25 \quad .129886+25$ $-.989764+25-.298524+28$ $-.549838+31 \cdot 194934+31$ $30^{\circ}$

$0100000+01 \quad .000000$

$.101974+01$.1163E3-01

$103989+01.240025-01$

$106043+01 \quad 371373-01$

$108137+01$. 510811-01

$.110269+01.658763-01$

$112441+01$-815669-01

$.114650+01.981992-01$

$116896+01 \quad 115821+00$

$.119178+01 \cdot 134483+00$

$.121495+01 \quad \cdot 154237+00$

$.133536+01 \quad .271371+00$

- $146100+01 \quad .424910+00$

$.158657+01 \quad .623876+00$

$.170349+01 \quad .878536+00$

$.179848+01.119994+01$

$185201+01-159889+01$

- $185201+01$ - $159889+01$

$.183636+01 \quad .208415+01$

$171377+01 \quad .265934+01$

$\begin{array}{ll}.143482+01 & .331817+01 \\ .938062+00 & .403754+01\end{array}$

$.151941+00 \quad .476793+01$

$-.998216+00 \quad .542135+01$

$-.257574+01 \quad .585743+01$

$-.462254+01 \quad .587039+01$

$-.708673+01.518191+01$

$-.980423+01 \quad .344908+01$

$-.124081+02 \quad .301777+00$

$-.142543+02-.457055+01$

$-.143607+02-.112629+02$

$-.114096+02-.194032+02$

$-.389141+01-.278220+02$

$.950566+01-.341808+02$

$.291411+02-.347103+02$

$.532363+02-.243320+02$

$0763516+02-0239913+01$

$.870744+02.489857+02$

$.722456+02 \quad .112885+03$

$.105895+02 \quad .179798+03$

$.345002+03-.846681+03$

$.165184+04 \quad .572854+04$

$.127488+05-.482630+05$

$-.505861+06 \cdot 179234+06$

$.359254+07 \quad .647923+07$

$.127587+09-.310891+08$

$.133025+10-.267648+10$

$.207375+10-.873217+11$

$.630673+11-.327704+13$

$.683728+14-.142358+15$

$.943832+16-.252614+16$

$.397576+18 \quad .666613+18$

$$
37.5^{\circ}
$$

$100000+01 \quad .000000$

$101801+01.141302-01$

$103620+01.290671-01$

$105458+01.448455-01$

$.107311+01.615009-01$

$.109177+01.790701-01$

.975904-01

$112943+01 \quad .117100+00$

$0114836+01 \quad 137638+00$

$.116733+01.159244+00$

$118629+01 \cdot 181958+00$

$.127976+01 \cdot 313595+00$

- $136645+01$. 479143+00

$143813+01 \quad .683551+00$

$.148357+01.930808+00$

$148800+01.122283+01$

$143293+010155787+01$

$129634+01 \quad 192846+01$

$105379+01.231879+01$

$.680682+00 \quad .270192+01$

$.156290+00 \quad .303701+01$

$-.530066+00 \quad .326754+01$

- $137052+01 \quad \cdot 332164+01$

$-.332543+01.256681+01$

$-.423536+01 \cdot 160541+01$

$-.487786+01 \quad .206798+00$

$-.502963+01-.157716+01$

$-.445375+01-0358054+01$

$-.295578+01-.549415+01$

$-.468263+00-.686146+01$

$.284504+01-.712231+01$ $.649023+01-.572745+01$

$.959591+01-.233541+01$

$.109818+02.292473+01$

$041085+010910036+01$

$.405857+01.147289+02$

$-.485116+01 \quad 1171054+02$

$-.154399+02 \quad .138266+02$ $.352504+02-.318118+02$ $-.121850+03 \cdot 315751+02$ $.286262+03.246430+03$ $.714413+03-.107588+04$ $-.294447+04-.407345+04$ - $217456+05-.476709+04$ $-.112172+06 \quad .340422+04$ $-.630212+06-.132005+06$

$-.252634+07-.336114+07$

$.165524+08-.265076+08$

$.206701+09 \cdot 164806+09$

$-.243169+10 \quad .751742+09$
45

.000000

$101595+01 \cdot 163619-01$

$103188+01.335494-01$

$104775+01.515878-01$

$.106354+01.705024-01$

$.107920+01.903181-01$

.111059+00

$111004+01 \cdot 132751+00$

$112513+01 \quad 155415+00$

$113995+01: 179075+00$

$115445+01.203753+00$

. $122045+01 \quad .343076+00$

$.127026+01 \quad .510240+00$

$.129522+01 \cdot 705740+00$

$128502+01 \cdot 927798+00$

$122814+01 \quad 117142+01$

$111267+010142738+01$

$927639+00.168134+01$

$665017+00$. $191326+01$

$.322278+00.209753+01$

$-.945930-01 \quad .220410+01$

.220118+01

$-.106664+01 \quad .205955+01$

$-.194085+01.129487+01$

$-.219057+010687583+00$

- $228534+01-0131460-01$

$-.201049+01-.725740+00$

$-152134+01-134236+01$

$-.798885+00-.174372+01$

$.610500-01-.182367+01$

$.907518+00-.152069+01$

$155613+01-0849408+00$

$183009+01 \quad .787847-01$

$161505+01.105855+01$

$.913632+00 \quad 182663+01$

$-121594+00.213210+01$

$-.119968+01.182716+01$

$-.196121+01 \quad .948998+00$ $.178275+01-.513342+00$ $-.201785+01-.479428+00$ $.248394+00 \cdot 205738+01$ $.190113+01-.186596+00$

$.714128+00-0176703+01$

. $323300+00-0101003+01$

$-.383674+00-191335+01$

$543630+00-185111+01$

$.185781+01-.535787+00$

$.733467+00 \quad 188952+01$

$0.50421+01-.598650-01$ 
RHOI THETA

$.00 \quad .100000+01$

$.02 .101443+01$

$.102869+01$

$-104275+0$

$-105656+01$

$.107009+01$

$.108331+0$

$.109617+01$

- $110862+0$

$.112062+01$

$113213+01$

-120806+0

$120731+01$

$116900+0$

$108743+0$

$.953336+00$

$.763292+00$

$\begin{array}{rr}1.00 & .517275+00 \\ 1.10 & .221086+00 \\ 1.20 & -.112292+00\end{array}$

$1.20-.112292+00$

$.30-.762011+00$

$1.50-.109192+0$

1.50 -.130302+01

$1.60-.130302+01$

$1.70-.140154+01$
$1.80-.136040+0$

$1.80-.136640+01$

.000000

176847-0

$01748-01$

$.756385-01$

$.118515+00$

$141267+00$

. $164910+00$

$.189453+00$

$.214906+00$

$.519531+00$

$.703602+00$

$.903774+00$

$111228+01$

$131617+0$

- $150697+01$

$166123+0$

$8923+0$

$.172780+01$

- $156770+0$

$.130926+01$

$965812+00$

$565275+00$

- $149150+00$

$1.90-.119347+01-.231426+00$

$2.00-.900424+00-.523642+00$

$2.10-.527958+00-.682837+00$

$2.20-.135761+00-.683750+00$

$2.30 \quad .207215+00-.529536+00$

$2.40 \quad 0437243+00-0255005+00$

$2.50 \quad .512073+00-744257-01$

$2.60 \quad .424377+00 \quad .381557+00$

$2.70 \quad .207358+00 \quad .591072+00$

$2.80 \quad-.711724-01 \quad .654101+00$

$2.90 \quad-.326684+00 \quad .563991+00$

$3.00-.483674+00$

$3.50 \quad .999905-01$

$4.00-.218410+00$

$4.50-.558352-01$

$5.00-.517307-01$

$5.50-.679421-01$

$6.50-.576053-01$

$7.00-.526816-01$

$7.00-.526816-01$

$7.50 \quad-.488796-01$
$8.00 \quad-.457747-01$

$8.50-.430626-01$

$9.00-.406279-01$

$.360592+00$

$.472332-02$

$.993987-01$

$.147044+00$

$.724167-01$

$.672066-01$

.683196-01

. $650358-01$

$.571703-01$

$.537426-01$
$.506142-01$

$.478200-01$ $60^{\circ}$

$.100000+01.000000$

$.101108+01 \quad 198904-01$

$.102172+01.404727-01$

$.103189+01 \quad .617442-01$

$104154+01$

$.617442-0$

. $106335+00$

$.105064+01$

$105916+01 \quad .129639+00$

$106704+01 \quad 153603+00$

ilorazator $\quad 178212+00$

$.108074+01 \cdot 203453+00$

$.108648+01 \quad+229307+00$

$.110242+01 \quad .366982+00$

$109341+01 \quad .515837+00$

$105496+01-670616+00$

$08797+000024347+00$

.070285+00

- 7 (c8587400

. $109394+01$

$1+00 \quad 119083+0$

$125055+01$

$.126642+0$

.123493+01

$-211562+00$ - $115668+0$

$-.372237+00 \quad 103688+01$

- $494065+00 \quad 005190+00$

(1)

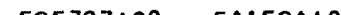

- $541594+00$

. $380624+00$

.245402+00

$2005+00 \quad 145177+00$

$.336258+00 \quad .837718-01$

$-.243313+00 \quad .592582-01$

$-.164751+00 \quad .647151-01$

$-.631827-01$

$-.671444-01$

$. .794229-01$
$-.932090-01$

$-.103604+00$

$-.891007-01$

$-.749050-01$

-.658583-01

$-.587417-01$

$-.530230-0$

$.483467-01$

$-.444434-01$

$-.411335-01$

$-.382898-01$

$-.358190-01$

-.336516-01

-.317343-01

$.179408+00$

- $183219+00$ $70^{\circ}$

$.100000+01 \quad .000000$

$100741+01$.214606-01

$101417+01.434163-01$

$102026+01.658462-01$

$102564+01.887273-01$

$103020+01$ 112035+00

$.103418+01: 135743+00$

$.103728+01: 159822+00$

$.103956+01: 184242+00$

$.104100+01.208971+00$

$.104157+01 \quad .233974+00$

$.103071+01.361797+00$

$.995993+00.490450+00$

$.614113+00$

$.851095+00 \quad .726620+00$

$.745231+00.821976+00$

$.622230+00 \quad 894945+00$

$.487972+00 \quad .941619+00$

$.349369+00 \quad .959889+00$

$.949748+00$

.913353+00

$-.215044-01.854822+00$

$-.110704+00 \quad .779790+00$

$-.177075+00 \quad .694764+00$

- $120337+00000035+00$

$-.242190+00 \quad .520751+00$

$-.245914+00 \quad .442725+00$

$-.235801+00 \quad .375650+00$

$-.216510+00.321167+00$

$-.192467+00 \quad .279344+00$

$-.167392+00 \quad .248556+00$

- $144014+00.228112+00$

- $123506+00$. $214327+00$

$-107586+00$ 0 $205321+00$

- $07586+00$. $205321+00$

-.959278-01 $\quad 199114+00$

$199114+00$
$.194224+00$

$189696+00$

$-.734511-01$

$.185036+00$

$.180089+00$

$.154611+00$

$134600+00$

. $119295+00$

$.107125+00$

$.972205-01$

.890010-01

$.820692-01$

$.761436-01$

$.710192-01$

$.665434-01$

$.626001-01$
$.590993-01$

$.542828-01-.231091-01$

$-.217828-01$ $80^{\circ}$

$.100000+01.000000$

$.100354+01.223563-01$

$.100631+01.449542-01$

$.100830+01.677607-01$

$.100949+01 \quad .907418-01$

$.100986+01.113862+00$

$.100942+01 \cdot 137086+00$

$.100814+01: 160377+00$

$100603+01: 183697+00$

$.100307+01.207009+00$

$.999272+00 \quad .230273+00$ $.967640+00 \quad .344526+00$

$.915655+00 \quad 451794+00$

$047520+00$

$.759756+00 \quad .627812+00$

$.663105+00 \quad .689772+00$

$.560035+00 \quad .731740+00$

$.455412+00 \quad .753397+00$

$.353863+00 \quad .755730+00$

-740868+00

$.175175+00 \quad .711800+00$

$.103231+00 \quad .672034+00$

$.445420-01.625237+00$

$-.045684-03 \quad .574901+00$

$-.341317-01 \quad .524075+00$

$-.565267-01 \quad .475193+00$

$-.699973-01.429992+00$

$-.765256-01 \quad .389521+00$

.780102-01 $0154223+00$

$.761241-01.324056+00$

$-.722347-01 \quad .298640+00$

$-.673774-010.277393+00$

-.620737-01 0250650+00

- $573770-01$. $259750+00$

$-.529309-01.232099+00$

$-.490308-01 \quad .221154+00$

-.456768-01 .211636+00

$-.428169-01$

$-.403769-01$

$-.321314-01$

-.270923-01

$-.235275-01$

$-.208412-01$

$-187325-01$

$-.156161-01$

$-.144276-01$

-.134115-01

$-.125322-01$

$-.117632-01$

$-110848-01$

- $195423+00$

$.164984+00$

$.143006+00$

$.126308+00$

$113170+00$

$446+00$

$937703-01$

$.801029-01$

$.746733-01$

699379-0

$.657709-01$

$.620752-01$

.000000

$99600+00 \quad .225616-01$

$.996406+00.675405-01$

$.993620+00 \quad 898862-01$

$.990050+00 \quad 112009+00$

$005703+00.134113+00$

. $134113+00$

$074725+00$ -

$.968119+00 \quad 198777+00$

$.960789+00 \quad .219753+00$

$.913931+00.318916+00$

$.852144+00.406153+00$

$778801+00 \quad 470925+00$

$.697676+00 \quad .535713+00$

$.612626+00 \quad .576042+00$

$.527292+00 \quad .600412+00$

$.444858+00 \quad .610142+00$

$.367879+00 \quad .607158+00$

$.598197+00 \quad .593761+00$

$.236928+00.572397+00$ 